$$
\angle A-S U B-93-229
$$

\title{
NONLINEAR RESPONSE OF PLAIN CONCRETE SHEAR WALLS WITH ELASTIC-DAMAGING BEHAVIOR
}

\author{
by \\ S. Yazdani and H. L. Schreyer \\ Departments of Civil and Mechanical Engineering \\ University of New Mexico \\ Albuquerque, New Mexico 87131
}

\begin{abstract}
This report summarizes the theoretical and computational efforts on the modeling of small scale shear walls. Small scale shear walls are used extensively in the study of shear wall behavior because the construction and testing of full size walls are rather expensive. A finite element code is developed which incorporates nonlinear constitutive relations of damage mechanics. The program is used to obtain nonlinear load-deformation curves and to address the initial loss of stiffness due to shrinkage cracking. The program can also be used to monitor the continuous degradation of the fundamental frequency due to progressive damage.
\end{abstract}

\section{DISCLAIMER}

\begin{abstract}
This report was prepared as an account of work sponsored by an agency of the United States Government. Neither the United States Government nor any agency thereof, nor any of their employees, makes any warranty, express or implied, or assumes any legal liability or responsibility for the accuracy, completeness, or usefulness of any information, apparatus, product, or process disclosed, or represents that its use would not infringe privately owned rights. Reference herein to any specific commercial product, process, or service by trade name, trademark, manufacturer, or otherwise does not necessarily constitute or imply its endorsement, recommendation, or favoring by the United States Government or any agency thereof. The views and opinions of authors expressed herein do not necessarily state or reflect those of the United States Government or any agency thereof.
\end{abstract}




\section{DISCLAIMER}

Portions of this document may be illegible in electronic image products. Images are produced from the best available original document. 


\section{TABLE OF CONTENTS}

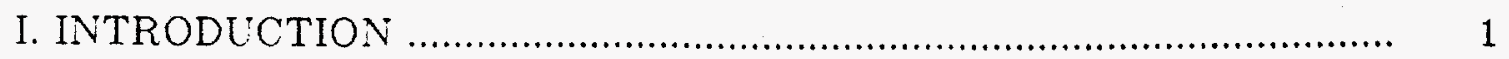

II. A CONTINUUM DAMAGE MECIIANICS MODEL ……....................... 3

III. THE GENERAL FORMULATION OF THE FEM PROGRAM …......... 9

IV. PROBLEM AND RESULTS …................................................... 13

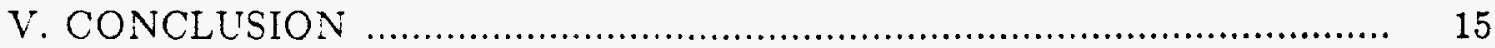

VI. REFERENCES, TABLES, AND FIGURES …….............................. 16

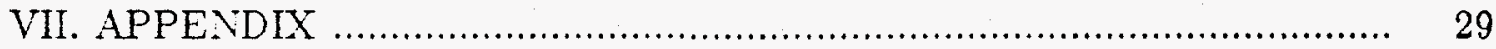




\section{Introduction}

Shear walls are important structural members which support gravity loads and can be designed to resist horizontal forces due to wind or seismic action. Since the full size construction and testing of shear walls are expensive, many studies have been carried on scaled down models. One such investigation as a part of an ongoing research program has been conducted by Endebrock, et al. (1985) at the Las Alamos National Laboratory. In their work, shear wall models with high şurface to volume ratios were used. The test results show that small scale models exhibit nonlinear behavior with an initial loss of stiffness and lower fundamental frequency than those computed with conventional linear elastic formulation or those observed in prototype walls.

The observed nonlinearities in the behavior of non-homogeneous cementitious materials arise from two distinct microstructural changes. One is the development of plastic flow along preferred dislocation planes under a high confining pressure. The second pattern involves the nucleation and propagation of microvoids and microcracks. Since the design and behavior of most conventional structural members, including shear walls, are under low or zero confining pressure it is plausible to assume that the nonlinear behavior of shear walls is strongly influenced by microcracking.

The presence of nonhomogeneities in the form of aggregate or reinforcement has microstructurally two conflicting effects. It is known for example that weak links develop at the interface of mortar and aggregate due to the accumulation of water lenses. These weak interface bonds are the sources of nucleation and propagation of microcracks. On the other hand, aggregate particles serve as crack arresters and help improve the strength and apparent ductility of mortar. The idea that aggregate particles act as energy barriers have been used to explain why high strength concrete behaves in a relatively more brittle fashion than normal strength concrete.

It is believed that reinforcement plays similar roles as manifested by numerous experiments. For most effective reinforcing action, it is essential that concrete and reinforcement deform together which in turn implies the necessity of developing strong bonds between them. The bond strength between the reinforcment and concrete is developed through (a) chemical adhesion, (b) natural roughness of the rebar, and (c) closely spaced rib-shaped deformation that provides a high degree of interlocking of the two materials. The majority of the bond strength develops through the interlocking of the two materials. The 
experimental investigations by Ervin and Jundi (1969) have shown that bonding of rebars is nonlinear and in particular looses stiffness with alternating loading. With this nonlinear characterization of the bond and the nonlinear behavior of concrete it is not surprising to see that a finite element program with a linear elastic material model overestimates that response stiffness. Since the reinforcement plays little role during the early stages of loading, continuum damage mechanics can be used to predict the associated anisotropic degradation in stiffness of the shearwalls.

Another aspect of the behavior that may warrant consideration is the shrinkage cracking of concrete. Shrinkage is caused by the evaporation of the free water over what is needed for the hydration of cement gel. The rate and completeness of drying depends on ambient temperature, humidity, and the surface area that is available for the heat outhux. The work of Troxell, el al. (1958) has shown that, with the geometry of the mold unchanged, a chief factor which determines the amount of final shrinkage is the water content of the fresh concrete. In their investigation the same aggregates were used for all tests, but in addition to and independently of water content, the amount of cement was also varied from four to eleven sacks per cubic yard of concrete. This very large variation of cement content had only very minor effects on the amounts of shrinkage, compared with the effect of water content. Similar findings were reported by Mckeen and Ledbetter (1970). On the other hand, Hanssen and Mattock (1966) investigated the shrinkage characteristics of structural menbers made from the same batch but with different surface areas and volumes. They considered the surface to volume ratio to be the design parameter and concluded that members with higher surface to volume ratios showed higher shrinkage deformations.

This study addresses the issues outlined above. A finite element program incorporating continuum damage mechanics is described which can predict the nonlinear load-deformation curve and changes in the fundamental requency of the siructure due to damage. The study also shows that if initial damage attributed to shrinkage cracking is taken into account, a good correlation with experimental data can be obtained. 


\section{A Continuum Damage Mechanics Model}

Continuous damage mechanics is concerned with the progressive weakening of solids due to the development of microcracks and microvoids. As the nucleation and growth of microcracks create voids in the system, the load carrying capacity and stiffness are reduced and material is termed damaged. There is a strong directionality associated with the process of damage in general. The detail of the formulation will not be given here as it has been already compiled (Yazdani and Schreyer, 1988).

Consider small and isothermal deformations and note that for small deformations the additive decomposition of tensors of any rank is permissible. Let $\mathbf{C}$ be the fourth-order material compliance tensor which is solely affected by the extent of microcracking. If the initial material compliance is denoted by $\mathbf{C}^{0}$ and the added flexibility is given by $\mathbf{C}^{c}$ the following relation holds:

$$
\mathbf{C}(k)=\mathbf{C}^{0}+\mathbf{C}^{\mathbf{c}}(k)
$$

where $k$ is a scalar parameter defining the extent of accumulated damage. The Cauchy stress tensor and the compliance of the material are related by means of Gibbs potential energy, G, as follows:

$$
\frac{\partial^{2} G(\boldsymbol{\sigma}, k)}{\partial \boldsymbol{\sigma} \partial \boldsymbol{\sigma}}=\mathbf{C}(k)
$$

The integration of Equation (2) with respect to $\sigma$ and the use of Equation (1) yields:

$\boldsymbol{\epsilon}=\frac{\partial G(\boldsymbol{\sigma}, k)}{\partial \boldsymbol{\sigma}}=\mathbf{C}^{0}: \boldsymbol{\sigma}+\mathbf{C}^{\boldsymbol{r}}(k): \boldsymbol{\sigma}+\boldsymbol{\epsilon}^{r}(k)$

where $\epsilon$ denotes the total strain tensor and $\boldsymbol{\epsilon}^{r}(k)$ arises as a variable of integration and is interpreted as the inelastic component of the deformation. If $\epsilon^{\prime}(k)$ is set to zero, the conventional definition of an elastic-perfectly damaging behavior is retained. This type of behavior is called elastic damage (Yazdani and Schreyer, 1988) to distinguish it from any inelasticity that may develop from inelastic fracturing. This inelastic feature may arise due to imperfect microcracking and crack tip process zones. To understand the structure of Equation (3) better, the rate form of this equation is needed: 


$$
\begin{aligned}
\dot{\boldsymbol{\epsilon}} & =\mathbf{C}: \dot{\boldsymbol{\sigma}}+\dot{\mathrm{C}}^{\mathrm{c}}: \boldsymbol{\sigma}+\dot{\boldsymbol{\epsilon}}^{r}(k) \\
& =\dot{\boldsymbol{\epsilon}}^{e}+\dot{\boldsymbol{\epsilon}}^{D}(k)+\dot{\boldsymbol{\epsilon}}^{r}(k)
\end{aligned}
$$

where $\dot{\epsilon}^{e}$ is the ratio of the elastic deformation in the absence of any further microcracking, $\dot{\epsilon}^{D}(k)$ is the rate of deformation due to damage, and $\dot{\epsilon}^{r}(k)$ defines the rate of inelastic strain tensor. In order to complete the theory two kinetic relations must be postulated and developed. One formulation is needed to describe the evolution of $\dot{\boldsymbol{\epsilon}}^{D}(k)$ and a second relation is required to define $\dot{\boldsymbol{\epsilon}}^{r}(k)$. The first task is the identification of a kinetic equation for $\dot{C}^{c}(k)$ satisfying the following relation

$\dot{\boldsymbol{\epsilon}}^{D}(k)=\dot{\mathrm{C}}^{c}(k): \sigma$

Following the approach by Ortiz (1985) the stress tensor and the associated rate of added fexibility tensor, $\dot{C}^{c}$, are decomposed into modes I and $I I$ of microcracking as follows:

$\dot{\epsilon}^{D}(k)=\left(\dot{C}^{c}{ }_{I}(k)+\dot{C}^{c}{ }_{I I}(k)\right):\left(\sigma^{+}+\sigma^{-}\right)$

where $\sigma^{+}$and $\sigma^{-}$are the positive and negative cones of the stress tensor and subscripts $I$ and $I I$ refer to modes $I$ and $I I$ of fracturing, respectively. Mode I refers to the cleavage type cracking and is shown in Figure 1a. Mode II is a more complicated type of cracking and involves crack sliding and surface opening simultaneously (Figure $1 \mathrm{~b}$ ). It is implicitly assumed that these modes are decoupled in the sense that $\sigma^{+}$leads only to mode I cracking and mode II is activated if $\boldsymbol{\sigma}^{-}$is nonzero and satisfies the loading condition. Two fourth-order response functions are further developed such that

$\dot{\mathbf{C}}_{I}^{c}=k \mathbf{R}_{I}$

and

$$
\dot{\mathrm{C}}_{I I}^{\mathrm{c}}=k \mathrm{R}_{I I}
$$

These tensorial functions determine the direction of incurring damage. Guided by experimental and other theoretical studies. the following form was 
adopted for $\mathrm{R}_{I}$ :

$\mathbf{R}_{I}=\frac{\boldsymbol{\sigma}^{+} \Theta \boldsymbol{\sigma}^{+}}{\boldsymbol{\sigma}^{+}: \boldsymbol{\sigma}^{+}}$

where the symbol $\theta$ denotes the tensor product and : indicates a $C_{12}$ contraction operation. The compressive mode of cracking or mode $I I$ is the result of simultaneous action of shear sliding of an existing inclined microcrack and the opening of crack sides. It is also understood that the mean normal pressure impedes the formation of mode II fracturing, while no damage can take place under purely hydrostatic pressure. To accomodate these features and to address the increase in the apparent Poisson's ratio that cementitious materials exhibit, $\mathbf{R}_{I I}$ is decomposed into two parts:

$\mathbf{R}_{I I}=\mathbf{R}_{I I}^{d}+\mathbf{R}_{I I}^{h}$

The first part is postulated to be:

$$
\mathbf{R}_{I I}^{h}=w \frac{\tilde{\boldsymbol{\sigma}} \otimes \tilde{\boldsymbol{\sigma}}}{\tilde{\boldsymbol{\sigma}}: \tilde{\boldsymbol{\sigma}}}
$$

where

$\overline{\boldsymbol{\sigma}}=\boldsymbol{\sigma}^{-}-\lambda I$

and where $\lambda$ is the maximum eigenvalue of $\boldsymbol{\sigma}^{-}$. The material parameter $w$ accounts for the relative strength of concrete in tension and compression. The form for $\mathbf{R}_{I J}^{h}$ is given by Yazdani and Schreyer (1988) to be:

$\mathbf{R}_{I I}^{h}=w \alpha H(-\kappa)(\mu \mathrm{I}-\mathbf{I} \otimes \mathbf{I}), \quad \mu \geq 1$

where $\alpha$ is a parameter that brings in the effect of lateral pressure on the stressstrain response of concrete and $\lambda$ and $H(\cdot)$ are the minimum eigenvalue of $\tilde{\sigma}$ and the Heaviside function, respectively. $\underline{I}$ is the fourth-order identity tensor and $\mu$ is a scalar coefficient which is determined from experimental observations. These formulations imply that damage occurs in the direction of minimum eigenvalues (maximum absolute values) of $\boldsymbol{\sigma}^{-}$and that no damage is predicted for a purely 
hydrostatic compression path. Yazdani and Schreyer also proposed the following simple evolution equation for the inelastic part of.the strain tensor:

$\dot{\boldsymbol{\epsilon}}^{\top}(k)=k w \mathbf{S}^{-}+k w \beta \mathbf{S}^{+}$

where $\beta$ is a scalar coefficient that is determined from experimental records and is greater than one for permanent deformation. The negative and positive cones of $\boldsymbol{\sigma}^{-d}$, the deviatoric component of $\boldsymbol{\sigma}^{-}$, are denoted by $\mathbf{S}^{-}$and $\mathbf{S}^{+}$, respectively. In this approach it is assumed that the inelastic damage is only associated with mode II cracking. If $\beta=1$ no inelastic volumetric deformation is predicted. A particular damage surface can be obtained by using the special forms of $R_{I}$ and $\mathbf{R}_{I I}$ and Equation 14 as follows:

$$
\begin{aligned}
\Psi(\boldsymbol{\sigma}, k)= & \frac{1}{2} \boldsymbol{\sigma}^{+}: \boldsymbol{\sigma}^{+}+\frac{w}{2} \boldsymbol{\sigma}^{-}: \frac{\tilde{\boldsymbol{\sigma}} \otimes \tilde{\boldsymbol{\sigma}}}{\tilde{\boldsymbol{\sigma}}: \tilde{\boldsymbol{\sigma}}}: \boldsymbol{\sigma}^{-}+w\left(\mathbf{S}^{-}+\beta \mathbf{S}^{+}\right): \boldsymbol{\sigma}+\frac{w}{2} \alpha \mu H(-\lambda) \boldsymbol{\sigma}: \boldsymbol{\sigma} \\
& -\frac{9 w \alpha}{2} H(-\lambda) P^{2}-\frac{1}{2} t^{2}(k)=0
\end{aligned}
$$

where $\mathrm{P}$ is the mean pressure and $\mathrm{t}(\mathrm{k})$ denotes the damage function. The damage function may also be called the critical stress (Ortiz, 1985). It is implicit in the formulation that the form for $t$ can be established from experimental data for any stress path. An exponential function proposed by Smith and Young (1955) is used in obtaining a continuous damage function as follows:

$\boldsymbol{\sigma}_{t}=f_{t} \frac{\boldsymbol{\epsilon}_{t}}{\boldsymbol{\epsilon}_{u}} \operatorname{EXP}\left(1-\frac{\boldsymbol{\epsilon}_{t}}{\boldsymbol{\epsilon}_{u}}\right)$

where $f_{t}$ and $\epsilon_{u}$ are the tensile strength and the associated insile strain of concrete, respectively. Since mode I cracking is assumed to be perfectly brittle, the damage parameter,$k$, can be interpreted as the added flexibility under uniaxial stress so that for any point on the stress-strain curve the following relation holds:

$$
\boldsymbol{\sigma}_{t}=\frac{\epsilon_{t}}{\frac{1}{E_{0}}+k}
$$


Equations (16) and (17) can be combined to yield the damage function. The limit damage surface is obtained by setting $t=f_{t}$. For biaxial stress paths this surface is shown in Figure 2 which also contains the average test values of Liu, et al. (1972) and Andenaes, et al. (1977).

It should be mentioned that the alteration of elastic properties and its effect on the structural stiffness is solely predicted by the elastic damage relations. For this reason, the finite element program is developed for the elastic-perfectly damaging material. The inclusion of the inelastic damage relation in the damage surface is for the completeness of the theory.

\section{Initial Stiffness and Shrinkage Cracking}

The theoretical and experimental investigations by Picket (1946) have shown that a reasonable agreement between measured shrinkage strains and values calculated from the diffusion equation can be achieved. Picket has suggested that shrinkage deformation of concrete follows approximately the laws of diffusion similar to those expressing the flow of heat. Hanssen and Mattock (1964) followed the approach by Pickett and produced experimentally obtained design curves as is shown in Figures $3 a$ and $3 b$. They concluded that although volume/surface ratio does not reflect perfectly variations of both sizes and shapes, nevertheless, the degree of correlation found between the theory and experiment is satisfactory for purposes of engineering modeling and design.

These investigations have not addressed the effect of shrinkage cracking on the material and structural stiffnesses. At the present time the material parameters obtained from the standard cylinder tests are used by analysts to calibrate the theoretical models. There are convincing experimental results to indicate that the use of values from cylinder tests (volume/surface area $=1.5$ ) may become inappropriate. One such attempt was made by Endebrock, et al. (1985) to analyze the deformation characteristics of model shear walls. The finite element prediction of the structural stiffness has overestimated the measured response by as much as three times. Such discrepencies are believed to lie in the use of inappropriate material parameters. The shear wall used by Endebrock, et al. (1985) is sketched in Figure 4 and has a volume to surface ratio of 0.47 . The design curves of Hannsen and Mattock (1966), Figures $3 \mathrm{a}$ and $3 \mathrm{~b}$, indicate that for this ratio, the shrinkage deformation is 1.4 times greater than that of standard cylindrical specimens after 50 days of casting. This can, at least partially, explain the observed discrepencies. 
To improve on this perceived problem, it is plausible to assume that shrinkage cracking has no preferential direction so that the degradation in the initial stiffness is isotropic. Let $\mathbf{C}^{0}$ be the initial compliance tensor to be defined as $\mathbf{C}^{0}=\left(\beta_{1}+\beta_{2}\right) \underline{I}-\beta_{2} I \otimes I \quad$ if $\operatorname{tr}(\epsilon) \geq 0$

where $\underline{I}$ and $I$ are the fourth order and second order identity tensors, respectively, and $\beta_{1}$ and $\beta_{2}$ denote material parameters to be determined from two different loading pathis. The effect of these parameters on the structural responses are illustrated and discussed in section IV of the report. 


\section{The General Formulation of the FEM Program}

It can be seen that the governing constitutive equations outlined in section II of this report are nonlinear and therefore an incremental approach should be taken in the solution algorithm. Let $K^{i-1}$ be the global stiffness matrix of the structure corresponding to the ith loading step assembled from the information obtained at the previous step $\mathrm{i}-1$. The governing matrix equation for the static problem is given by

$$
\left[K^{i-1}\right]\left[\Delta u^{i}\right]=\left[\Delta P^{i}\right]
$$

where $\Delta u^{i}$ is the global incremental nodal displacement vector at the ith step and $\Delta P^{i}$ denotes the incremental loading vector also at the ith step. Equation 19 yields the nodal displacement vector, $\Delta u^{i}$, which can be used to obtain the corresponding incremental nodal strain vector, $\Delta \epsilon^{i}$, for each element as follows:

$\left[\Delta \epsilon^{i}{ }_{\text {elem }}\right]=\left[B_{\text {elem }}^{i}\right]\left[\Delta u^{i}{ }_{\text {elem }}\right]$

where $[B]$ is the strain-displacement matrix. Since the stiffness terms are computed numerically at the Gauss points, the program was modified to interpolate strains over the element using the element shape functions, $N$. The resulting expressions were evaluated at Gauss points:

$\left.\left[\Delta \epsilon^{i}{ }_{\text {elem }, k}\right]=\mid N_{\text {elem }}\right]\left[\Delta \epsilon_{\text {elem }}^{i}\right]$

where $\Delta \epsilon^{i}{ }_{\text {elem,k }}$ denotes the incremental strain vector corresponding to an element "elem" and a Gauss point " $k$ ". The strains at the Gauss points are desired because damage is computed at Gauss points and the damage subroutine is a strain driven algorithm. Let $A^{T}$ be the transformation matrix which can be used to transform a given matrix, $M$, to a diagonal matrix , $D$, as follows:

$\left.\left.[D]=\left[A^{T}\right] \mid M\right] \mid A\right]$

Such an operation can be done with respect to the components of the strain tensor to obtain the corresponding eigenvalues to be used in the damage subroutine. With this, the following subsection explains the structure of the solution algorithm used in the numerical integration of the anisotropic damage model. 


\section{The structure of the damage solution algorithm}

A numerical code for solving nonlinear constitutive relations is presented in this section. The code applies to an elastic-damaging material where the damage function has both hardening and softening features. The continuous alteration of elastic properties is a major feature of damage theory. For a given load increment, and after solving Equation 21, the governing set of equations that need to be solved are:

$$
\begin{aligned}
& \epsilon=\epsilon_{0}+\Delta \epsilon \\
& \sigma=D: \epsilon^{e} \\
& \epsilon^{e}=\epsilon-\epsilon^{* D} \\
& \epsilon^{* D}=\epsilon^{*} D+\Delta \epsilon^{D} \\
& \Delta \epsilon^{D}=\Delta k \frac{\partial \Psi}{\partial \sigma} \\
& k=k_{0}+\Delta k \\
& \Psi(\sigma, k)=0
\end{aligned}
$$

where $\epsilon^{* D}$ represents the components of the total strain tensor, $\epsilon$, due to elastic damage. The elastic strain tensor for an undamaged material is denoted by $\epsilon^{e}$ and subscript 0 refers to the previous time step as before. An increment is indicated by $\Delta$ and $\mathrm{k}$ is a scalar measure of cumulative damage. The damage surface is given by $\Psi$, and $\sigma$ and D represent the updated stress and stiffness tensors, respectively.

The underlining problem is that given an increment in total strain obtained form the previous step, $\epsilon^{\ell}, \epsilon^{* D}, \sigma, D$ and $\Delta k$ are unknown. An iterative approach for snlving Equations 23 is explained as follows:

Step 1: Enter the damage subroutine with an increment in total strain.

Step 2: Assume step is elastic.

$$
\begin{aligned}
& \Delta \epsilon^{e}=\Delta \epsilon \\
& I=0(I=\text { iteration count })
\end{aligned}
$$

Step 3: Adjust the strain increment for the particular path chosen, compute the increment in stress, and upriate the stress. 


$$
\begin{aligned}
& \Delta \sigma=D: \Delta \epsilon^{e} \\
& \sigma_{I}=\sigma_{I-1}+\Delta \sigma \\
& I=I+1
\end{aligned}
$$

Step 4: Compute the damage function and check the damage condition.

(a) If $|\Psi|<\bar{\epsilon}$ and $I=1$ (i.e. the first step), then the solution is elastic and the stress $\sigma$ is correct. Go to step 7 .

(b) If $|\Psi|<\bar{\epsilon}$ and I $>1$, where $\bar{\epsilon}$ is a specified tolerance, then a damage solution has been obtained and the stress $\sigma$ is correct. Go to step 7 .

(c) Else, go to step 5

Step 5: For $\mathrm{I}=1$, prescribe an initially small increment of $\Delta k$.

For I $>1$, use the Secant Method:

$$
(\Delta k)_{I}=-(\Delta k)_{I-1} \frac{\Psi_{I}}{\Psi_{I}-\Psi_{I-1}}
$$

Step 6: Calculate the added flexibility, increments of elastic and inelastic damage strains and update all variables.

$$
\left(\Delta C^{c}\right)_{I}=(\Delta k)_{I} R
$$

where $C^{c}$ is the added flexibility tensor and $\mathrm{R}$ is the fourth order response tensor determining the direction of incurring damage given by Fquation (20). Then

$$
\begin{aligned}
& \left(C^{c}\right)_{I}=\left(C^{c}\right)_{I-1}+\left(\Delta C^{c}\right)_{I} \\
& C=C_{I-1}+\left(C^{c}\right)_{I}
\end{aligned}
$$

where $\mathrm{C}$ is the current compliance tensor.

$$
\begin{aligned}
& D=C^{-1} \\
& \left(\Delta \epsilon^{D}\right)_{I}=\left(\Delta \epsilon^{D}\right)_{I-1}+(\Delta k)_{I}\left(\frac{\partial \Psi^{\prime}}{\partial \sigma}\right)_{I} \\
& \left(\Delta \epsilon^{e}\right)_{I}=\left(\Delta \epsilon^{e}\right)_{I-1}-(\Delta k)_{I}\left(\frac{\partial \Psi^{\prime}}{\partial \sigma_{I}}\right)_{I}
\end{aligned}
$$




$$
k=k_{0}+(\Delta k)_{1}
$$

Go to step 3.

Step 7: Update elastic, damage and total strain.

$$
\begin{aligned}
& \epsilon^{e}=\epsilon^{e}{ }_{0}+\Delta \epsilon^{e} \\
& \epsilon^{* D}=\epsilon^{*} D+\Delta \epsilon^{D} \\
& \epsilon=\epsilon^{e}+\epsilon^{*} D
\end{aligned}
$$

Step 8: Return to the main program for another load increment.

The plain stress condition of shear wall problems is handled during each loading increment in the damage subroutine. As was explained in step 3 before, the strain increment vector is adjusted for a particular path. For the plain stress path, the increment in the third normal strain component is:

$$
\Delta \epsilon^{e}{ }_{33}=-\left[D_{3311} \Delta \epsilon_{11}^{e}+D_{3322} \Delta \epsilon_{22}^{e}\right] / D_{3333}
$$

where $D_{3311}, D_{3322}$, and $D_{3333}$ represent the components of the updated stiffuess tensor, respectively. The components of the elastic incremental strain vector are given by $\Delta \epsilon^{e}{ }_{11}, \Delta \epsilon_{22}^{e}$, and $\Delta \epsilon^{e}{ }_{33}$. 


\section{Problem and results}

The finite element program developed was used to obtain the theoretical response features of the model shear wall used by Endebrock et al. (1985). Figures 5 and 6 show different mesh sizes, boundary conditions, loading functions, and the resulting load-deformation curves for the convergence study. It was determined that with 24 elements, convergence is sufficiently achieved and that this mesh arrangement would be used for the other examples. The material parameters for Figure 6 are given in Table 1. It should be mentioned that for a monotonic convergence to the damage surface in the constitutive equation subroutine, the strain increment of the order E-06 to E-05 should be used. This can be achieved by specifying small increments in the loading function. Loading increments of $5 \mathrm{E}-04$ kips to $5 \mathrm{E}-03$ kips were used and the resulting loaddeformation curves were practically identical.

For the initial cracking and its effect on the material stiffness the following modification of Equation 18 is considered. Let $\mathrm{E}$ and $v$ denote the Young's modulus and Poisson's ratio, respectively, of a material obtained from standard cylinder tests. For the first load increment when $\operatorname{tr}(\Delta \epsilon) \geq 0$, define two other parameters $\alpha_{1}$ and $\alpha_{2}$ such that $\beta_{1}=\alpha_{1} \frac{1}{E}$ and $\beta_{2}=-\alpha_{2} \frac{\nu}{E}$. For a special condition where $\alpha_{1}$ and $\alpha_{2}$ are both one, the conventional isotropic compliance tensor corresponding to the standard cylinder parameters are obtained.

Figure 7 shows the load-deformation curve and the nonlinear character that is associated with progressive damage. The linear elastic response also obtained from the program and the experimentally obtained values for the initial stiffness by Endebrock, et al. (1985) is also plotted. The material parameters for this example are tabulated in Table 2. It should be mentioned that a significant reduction in the material stiffness and its subsequent effect on the nonlinear load-deformation curve becomes only noticeable when material elements are strained beyond the limit state into the softening regime. A real structure can not sustain such a degree of distress and the role of reinforcing steel becomes important. Steel reinforcement was not modeled in this analysis. Figure 7 also illustrates the evolution of the damage and its effect on the response.

The variation of the initial stiffness due to varying values of $\alpha_{1}$ is plotted in Figure 8 where the material parameters of Table 3 are used. It was found that the response is more sensitive to the variation of $\alpha_{1}$ when the mesh arrangement is more coarse. The reason is that the value of $\alpha_{1}$ is implemented only under the 
condition when the trace of the total strain increment is positive. It was also found that the variation of the second parameter, $\alpha_{2}$, keeping $\alpha_{1}$ constant, does not introduce any noticeable change in the load-deformation curve.

The changes of the fundamental frequencies of the structure due to progressive damage can be obtained by solving the characteristic equation $\operatorname{det}\left[K-M \omega^{2}\right]=0$ where global stiffness and mass matrices are given by $\mathrm{K}$ and $M$, respectively, and $\omega$ denotes a natural frequency of the system. The eigenvalues were, obtained at each load level using an eigenvalue solver program available at the University of New Mexico. The fundamental frequency is plotted with respect to the applied loading in Figure 9 for the material parameters of Table 2, and the plot shows that the eigenvalue decreases with progressive degradation of the structural stiffness. This finding is consistent with the experimental work of Endebrock, et al. (1985) where the fundamental frequencies of several shear walls are found to decrease with progressive damage. 


\section{Conclusion}

A finite element program was developed. to incorporate the nonlinear material behavior due to progressive damage. The program requires a large amount of memory since the stiffness and flexibility matrices, the stress vector, the critical stress, and the cumulative damage parameter, among others, are stored for each Gauss point of every element. This is, however, a problem with most nonlinear codes and is not exclusive to the damage mechanics model.

A simple approach to the loss of stiffness due to shrinkage cracking is also outlined and its effect on the structural response is shown. With the lack of experimental investigations on the pattern of shrinkage cracking, an isotropic degradation of the initial stiffness is suggested. The use of initial damage, however, can explain the discrepancy in observed initial elastic response of two different sizes of models for shear walls.

The last part of the study was concerned with the changes in the fundamental frequencies with progressive damage. The reduction in natural frequency with the load is consistent with experimental observations.

This report shows that continuum damage mechanics is a potentially useful framework for reflecting the effects of initial shrinkage cracks and the reduction in natural frequencies of loaded concrete members. Although the development of such models is relatively new, there is a significant potential for accurately reflecting the behavior of concrete structures that display damage. However, for a direct correlation between theoretical and experimental data, the effects of reinforcing steel should be incorporated in the numerical simulation of the response of the shear walls.

\section{Acknowledgment}

This investigation was supported financially by the Los Alamos National Laboratory. 


\section{References}

- Andenaes, E., K. Gerstle and H.Y. Ko (1977), "Response of Mortar and Concrete to Biaxial Compression," J. Engng. Mech. Div., ASCE, Vol. 103, p.515.

- Endbrock, E.G., R.C. Dove, and W.E. Dunwoody (1985), "Analysis and Tests on Small Shear Walls," Report no. LA-10433-MS

- Ervin, S. P. and N. Jundi (1969), "Pullout Bond Stress Distribution Under Static and Dynamic Repeated Loadings," ACI, Vol. 66, No. 28, pp. 377.

- Hanssen, T.C. and A.H. Mattock (1966), "The Influence of Size and Shape of Member on the Shrinkage and Creep of Concrete," ACI Journal, Vol. 63, p.26.

- Liu, T.C.Y., A.H. Nilson and F.O. Slate (1972), "Stress-Strain Response and Fracture of Concrete in Uniaxial and Biaxial Compression", Proc. ACl, Vol. 69, No. 5 , p. 291

- Mckeen R. G. and W. B. Ledbetter (1970), "Shrinkage-Cracking Characteristics of Structural Light Weight Concrete," ACI, Vol. 69, No. 44, pp. 769.

- Picket, G. (1946), "Shrinkage Stresses in Concrete," ACI Journal, Vol. 42, no. 3, p.165.

- Smith, G.M. and L.E. Young (1955), "Ultimate Theory in Flexure by Exponential Function", Proc. ACI, Vol. 52, No. 3, p. 349

- Troxell, G. D., G. E. Raphael, and II. E. Davis (1958), "Long-Time Creep and Shrinkage Tests of Plain and Reinforced Concrete," ASTM Proceedings, Vol. 58, pp. $1101-1120$.

- Yazdani, S. and H.L. Schreyer (1988), "An Anisotropic Damage Model for Concrete with Dilatation," Mechanics of Materials, to appear. 


\begin{tabular}{|c|l|}
\hline Parameters & \multicolumn{1}{|c|}{ Values } \\
\hline $\mathrm{E}$ & $4000 \mathrm{ksi}$ (Young's modulus) \\
$\nu$ & 0.20 (Poisson's ratio) \\
$f_{c}$ & $4 \mathrm{ksi}$ (Uniaxial compressive strength) \\
$f_{t}$ & $.5 \mathrm{ksi}$ (Uniaxial tensile strength) \\
$\alpha$ & 0.00112 \\
$w$ & 0.0067 \\
$\mu$ & 1 \\
$\alpha_{1}$ & 1.0 \\
$\alpha_{2}$ & 1.0 \\
\hline
\end{tabular}

Table 1. Material parameters for the results shown in Figure 6. 


\begin{tabular}{|c|l|}
\hline Parameters & \multicolumn{1}{|c|}{ Values } \\
\hline $\mathrm{E}$ & $4000 \mathrm{ksi}$ (Young's modulus) \\
$\nu$ & 0.20 (Poisson's ratio) \\
$f_{c}$ & $4 \mathrm{ksi}$ (Uniaxial compressive strength) \\
$f_{t}$ & $.5 \mathrm{ksi}$ (Uniaxial tensile strength) \\
$\alpha$ & 0.00112 \\
$w$ & 0.0067 \\
$\mu$ & 1 \\
$\alpha_{1}$ & 0.80 \\
$\alpha_{2}$ & 1.0 \\
\hline
\end{tabular}

Table 2. Material parameters for the results shown in Figures 7 and 9. 


\begin{tabular}{|c|l|}
\hline Parameters & \multicolumn{1}{|c|}{ Values } \\
\hline $\mathrm{E}$ & $4000 \mathrm{ksi}$ (Young's modulus) \\
$\nu$ & 0.20 (Poisson's ratio) \\
$f_{c}$ & $4 \mathrm{ksi}$ (Uniaxial compressive strength) \\
$f_{t}$ & $.5 \mathrm{ksi}$ (Uniaxial tensile strength) \\
$\alpha$ & 0.00112 \\
$w$ & 0.0067 \\
$\mu$ & 1 \\
$\alpha_{1}$ & Variable \\
$\alpha_{2}$ & 1.0 \\
\hline
\end{tabular}

Table 3. Material parameters for the results shown in Figure 8. 


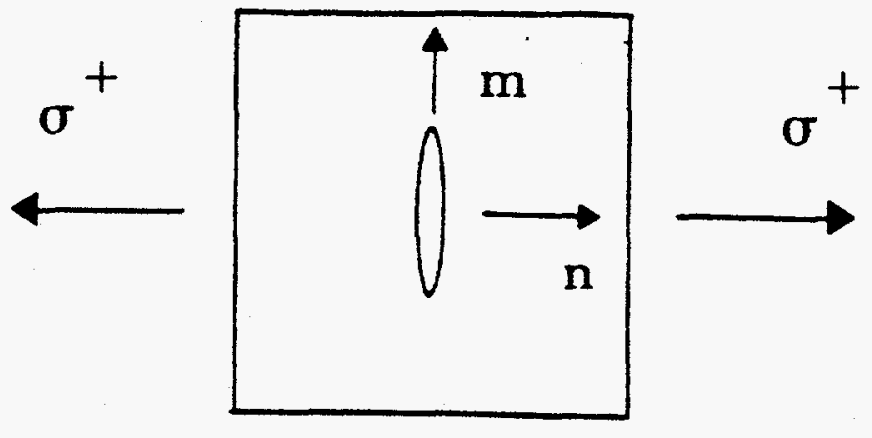

(a) Mode I

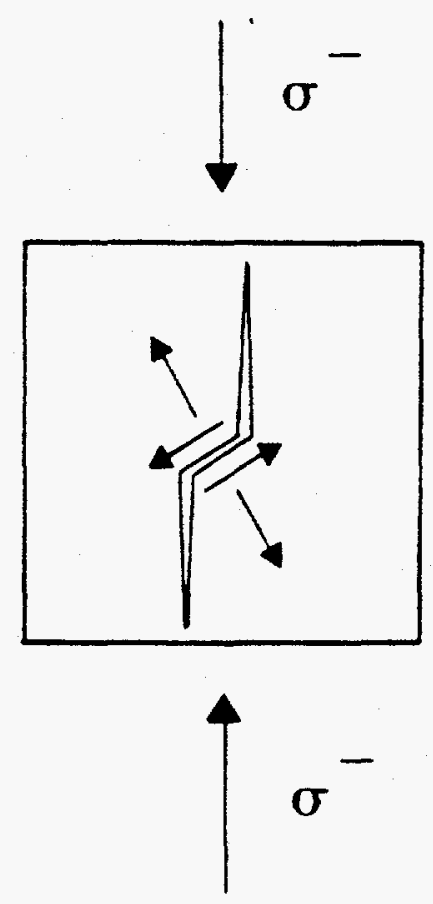

(b) Mode II

Figure 1. Schematic representation of crack opening in modes I and II. 


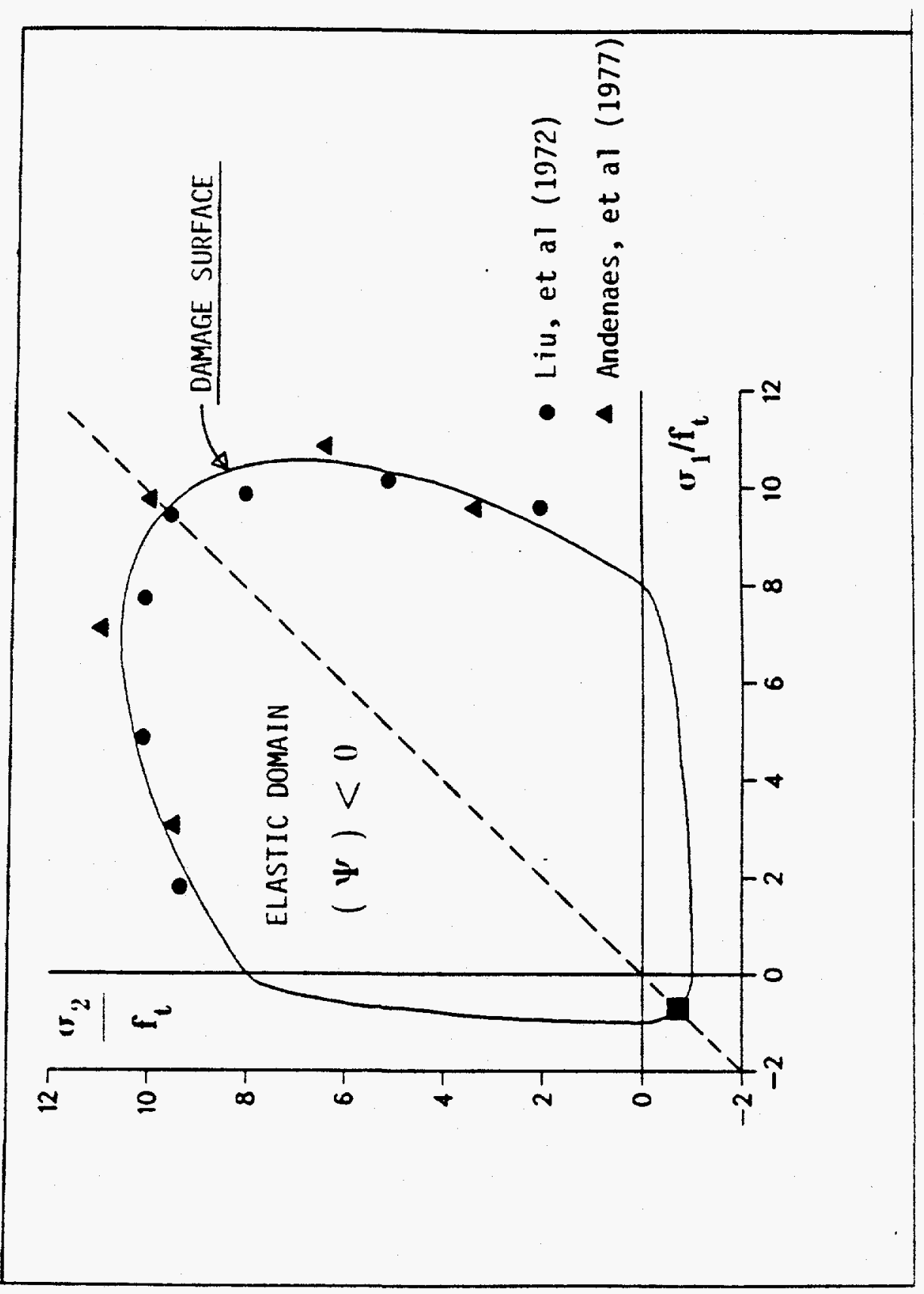

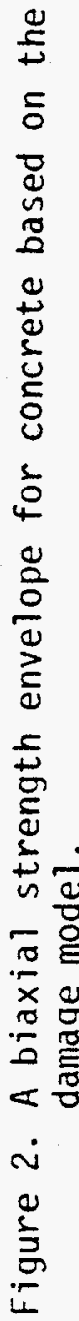




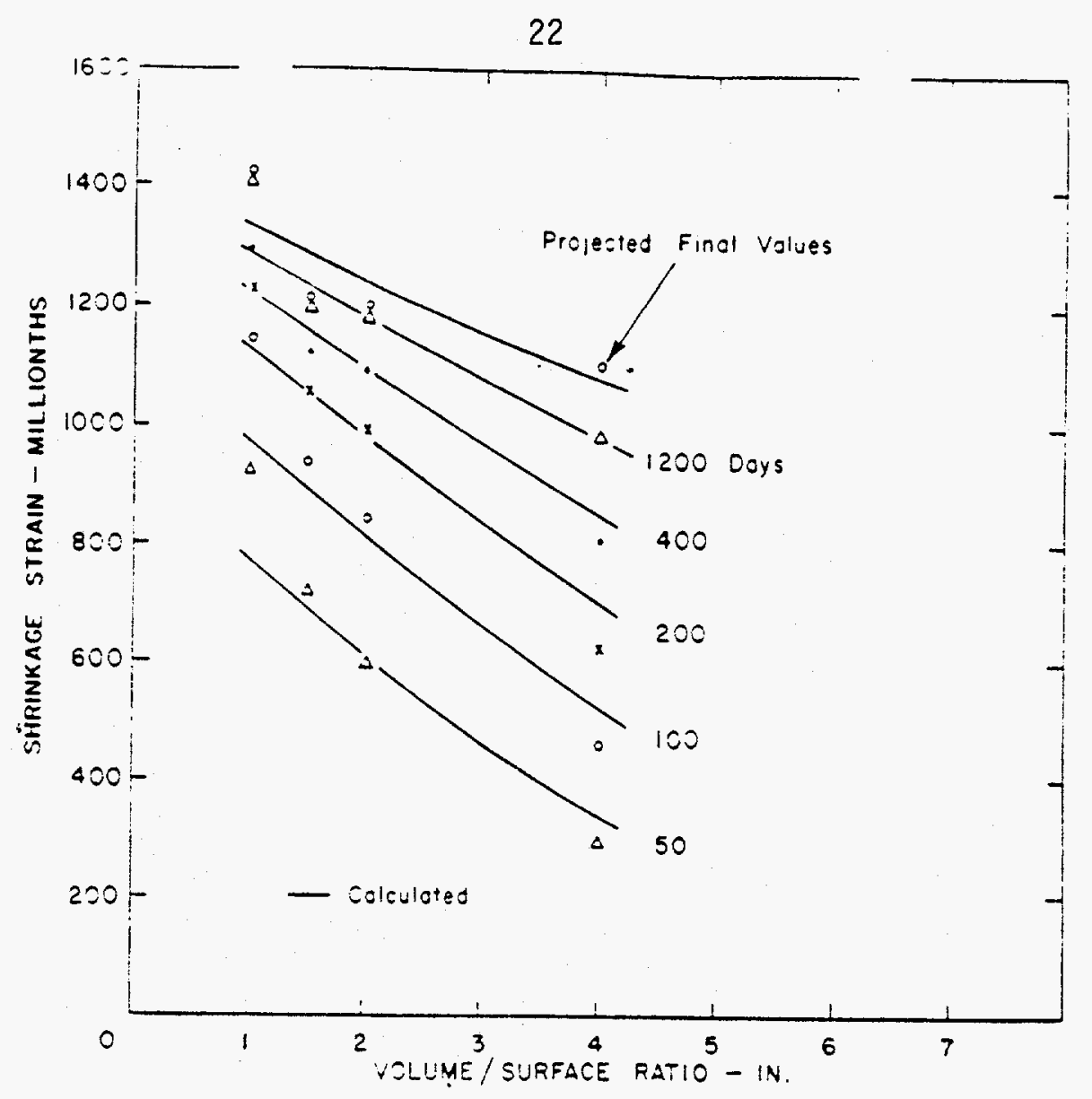

(a) Sand-stone aggregate concrete

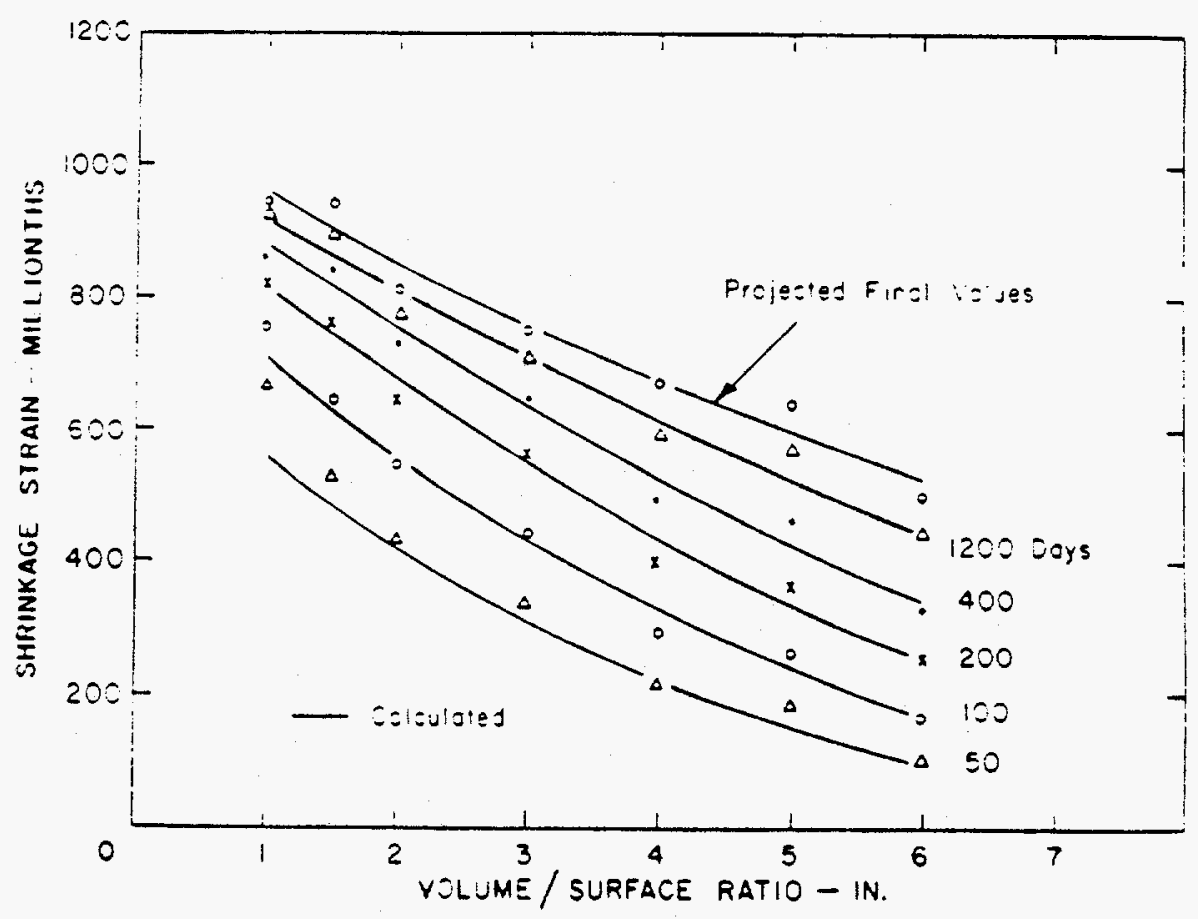

(b) Elgin gravel aggregate concrete

Figure 3. Variation of shrinkage with volume/surface ratio, at different ages (Hanssen and Mattock, 1966). 


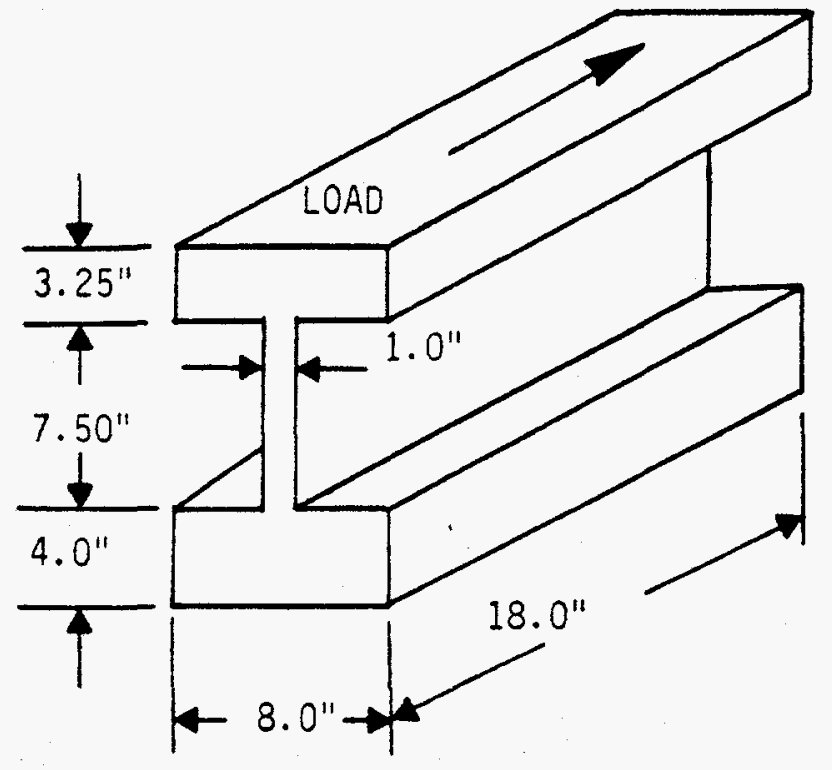

Figure 4. A schematic representation of the one-story model shear wall used by Endebrock, et al. (1985). 


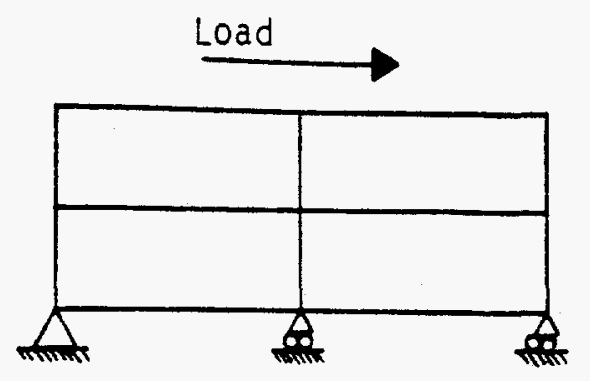

(a) 4-Element Mesh

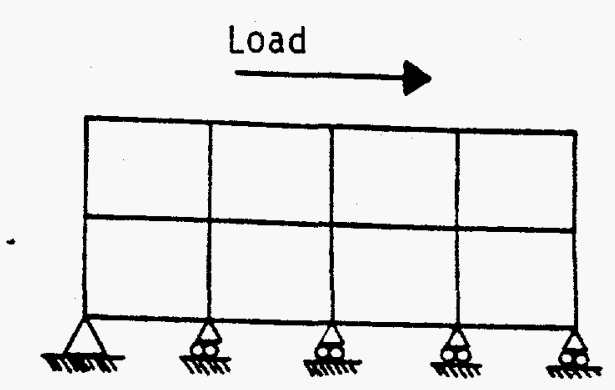

(b) 8-Element Mesh

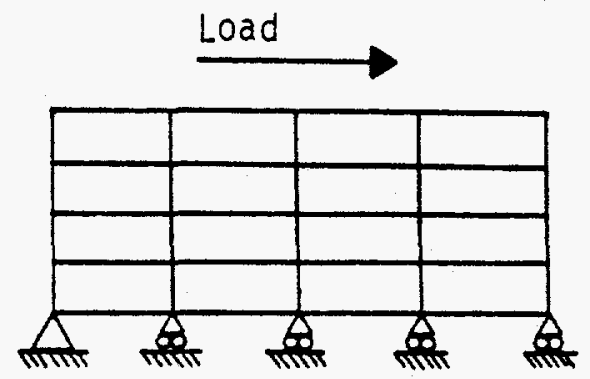

(c) 16-Element Mesh

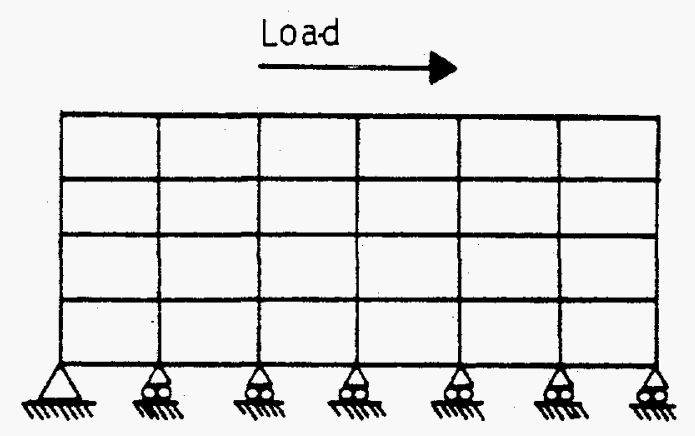

(d) 24-Element Mesh

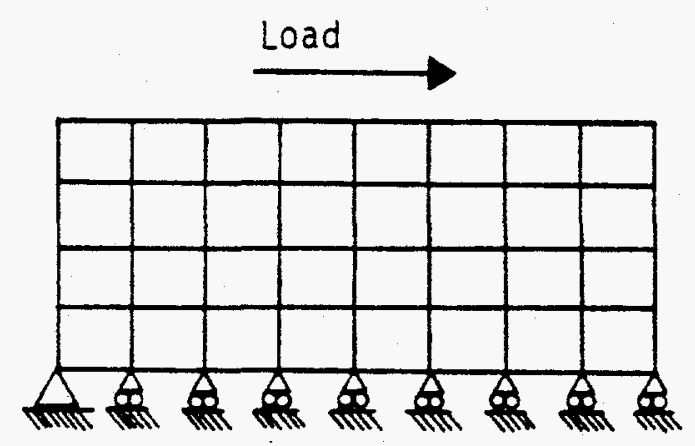

(e) 32-Element Mesh

Figure 5. A schematic representation of mesh arrangement, loading, and boundary conditions. 


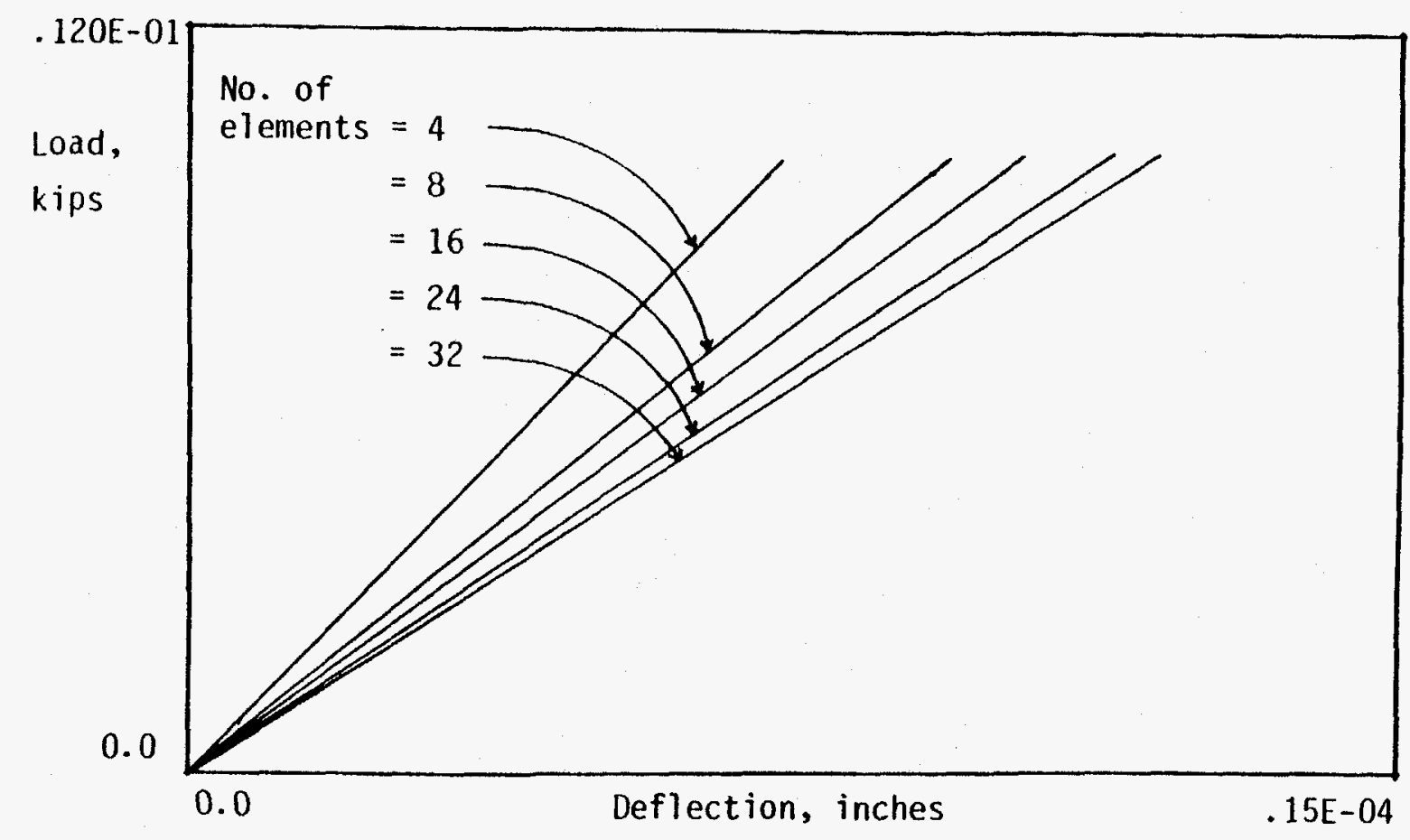

Figure 6. Load-deflection curves for a convergence study based on the mesh arrangements of Figure 5. 


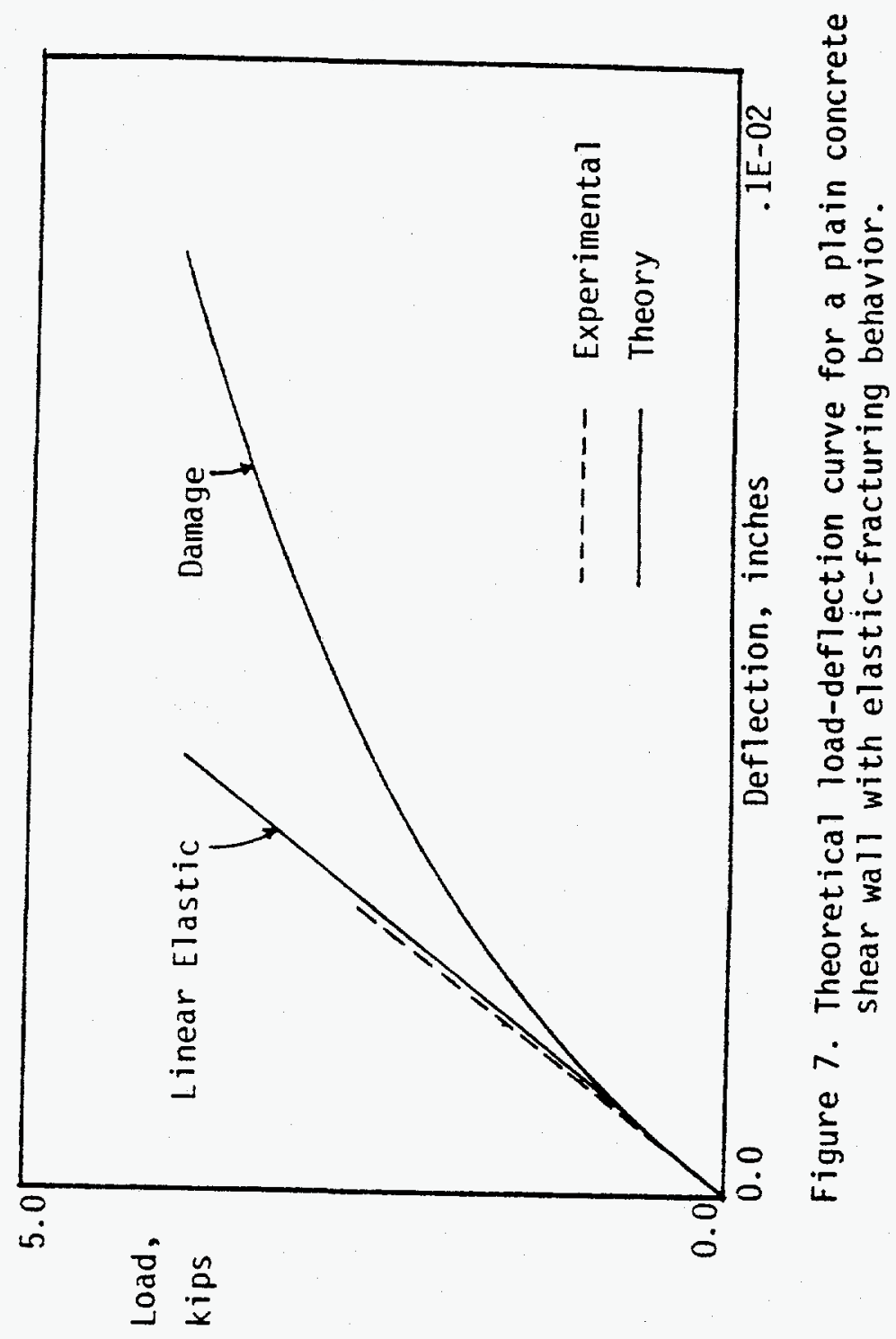




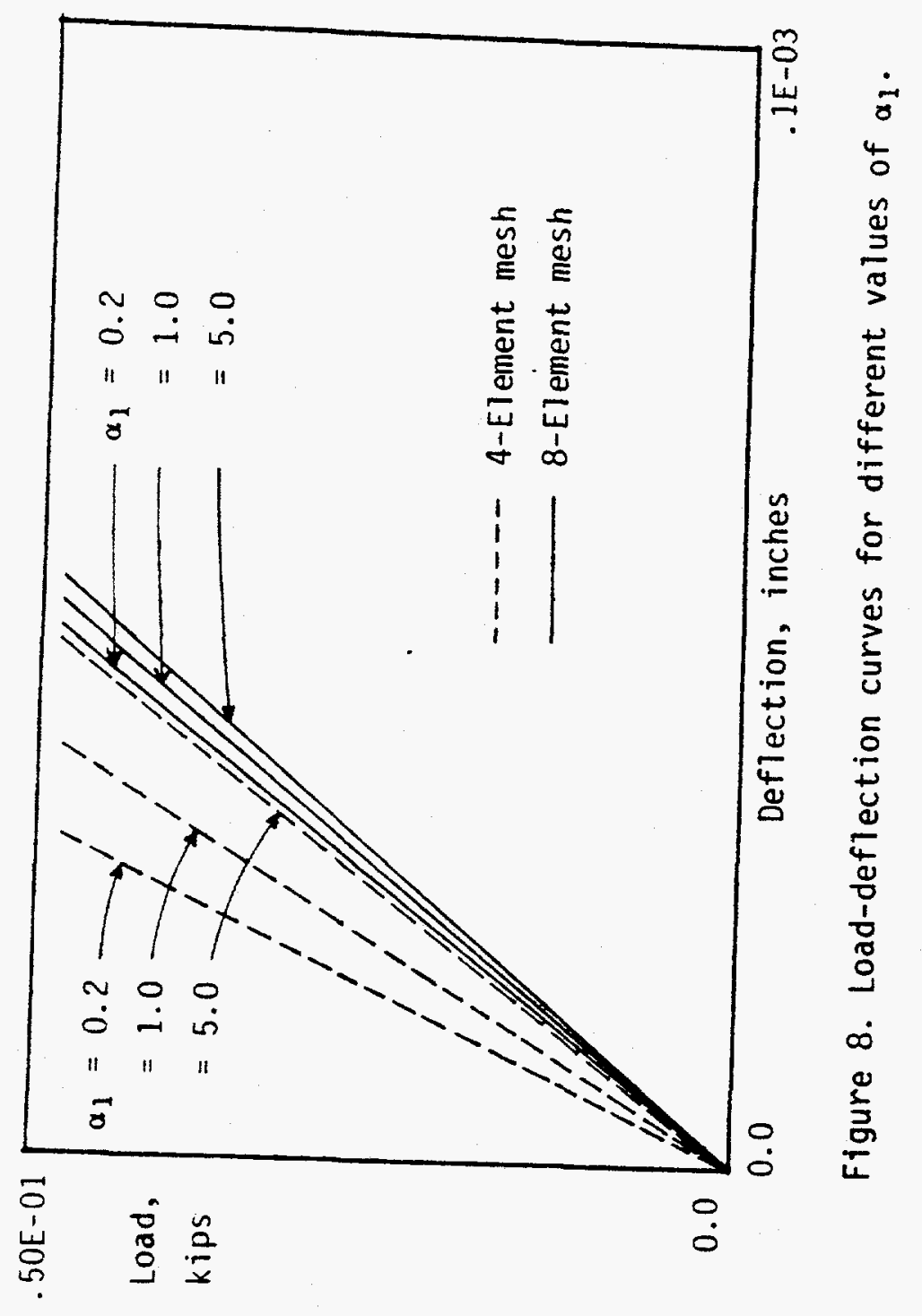




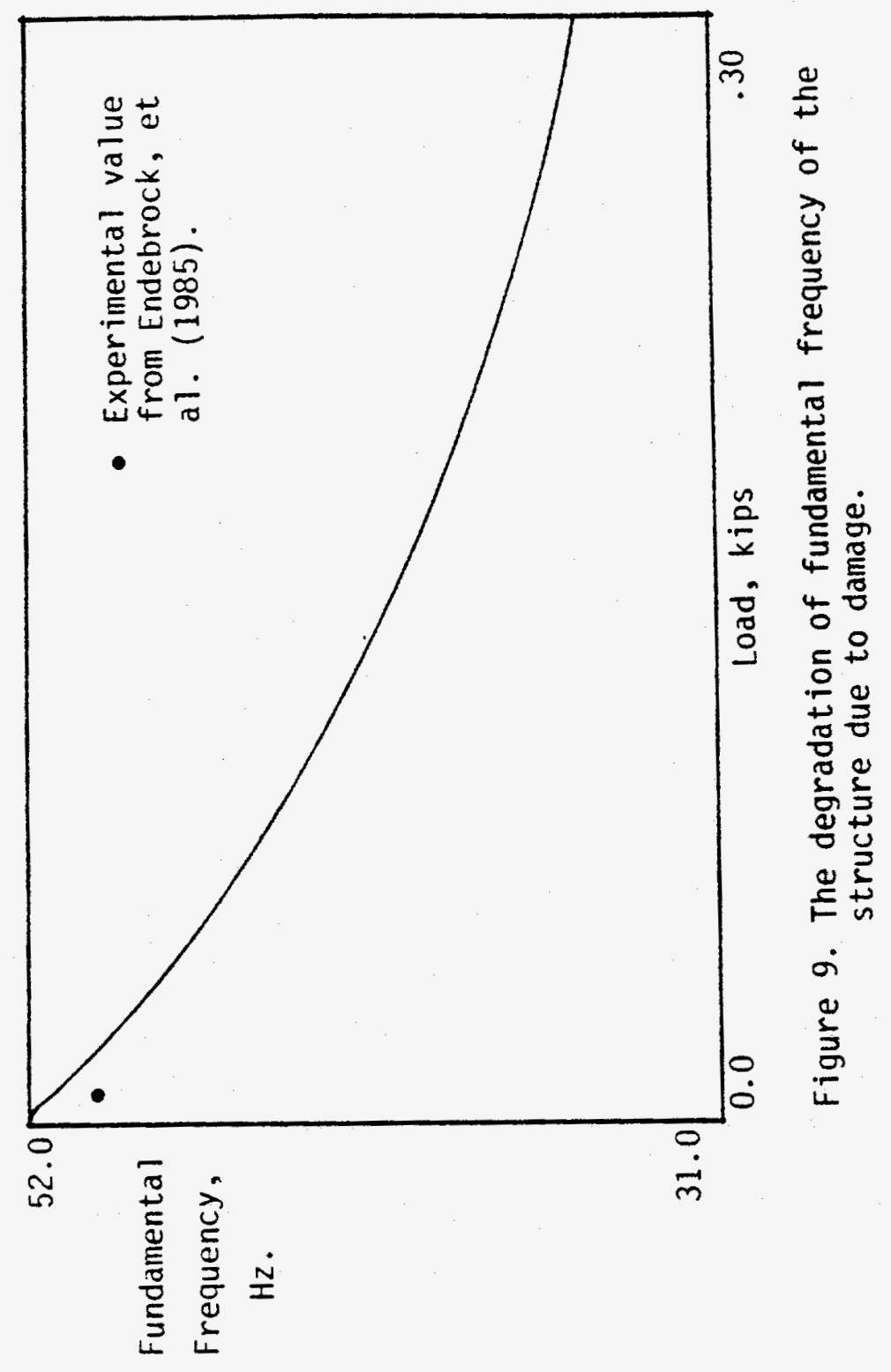




\section{Appendix}

The listing of the finite element program together with a simple flow chart representation and a sample of an input data is given in this section. The program consists of a driver and twenty one subroutines. Some of the subroutines are taken from a graduate level finite element class taught by Drs. Roy Johnson and Walter Gerstle in the department of Civil Engineering at the University of New Mexico. These include subroutines input, stif, stifQ4, elast, $\mathrm{n} 4, \mathrm{nn} 4$, disout, recovr, jacob, mult, matadd, aeqnum, band, assmbl, and solver of which the first eight were modified to be compatible with the nonlinear character of the problem. The remaining subroutines were developed by the authors and include programs eldam, damage, matinv, principal, and principal1. The latter two use an eigenvalue solver subroutine, dsyev, available at the University of New Mexico. 


\section{Flow chart of the program:}

Program main General control.

Call input

Reads in all data.

Call aeqnum

Assigns equation no. to the degrees of freedom.

Call band

Determines the band width of the stiffness matrix.

-[A]- Start the main do-loop:

Call stif

Call stifQ4

Call elast

Call nn4

Call n4

Call jacob

Call stdisp

Call mult

Call matadd

Call assmbl

Call principal1

Call solver

Call disout

Call recovr

Call nn 4

Call n4

Call jacob

Call stdisp

Call mult

Call eldam

Call damage

Call principal

Call matinv
Develops updated stiffness matrix.

Calculates the stiffness of a 4-noded elemeni.

Sets up the initial material stiffness.

Specifies local coordinates.

Computes shape functions and derivatives.

Forms the Jacobian and its inverse.

Sets up strain-displacement relationships.

An algorithm for multiplication of matrices.

An algorithm for addition of matrices.

Assembles the stiffness matrix.

Finds frequencies (normally first).

Solves the system of equations.

Obtains displacement increments.

Obtains strains from displacements.
Increment of strains are avialable at this pt.

Sets up the data for the damage relations.

Determines damage parameter and damage strain.

Finds eigenvect. for transf. to global coord.

Obtains stiffness from flexibility. 
A sample output for the shear wall shown on page 32 :

'Compression Member' $15,8,1,2,20, .005$

$1,1,2,5,4,0,0,0,0,4,100$

$2,2,3,6,5,0,0,0,0,4,100$

$3,4,5,8,7,0,0,0,0,4,100$

$4,5,6,9,8,0,0,0,0,4,100$

$5,7,8,11,10,0,0,0,0,4,100$

$6,8,9,12,11,0,0,0,0,4,100$

$7,10,11,14,13,0,0,0,0,4,100$

$8,11,12,15,14,0,0,0,0,4,100$

$1,0.0,0.0,20$

$2,3.75,0.0,80$

$3,7.5,0.0,70$

$4,0.0,4.5,20$

$5,3.75,4.5,80$

$6,7.5,4.5,90$

$7,0.0,9.0,20$

$8,3.75,9.0,80$

$9,7.5,9.0,90$

$10,0.0,13.5,20$

$11,3.75,13.5,80$

$12,7.5,13.5,90$

$13,0.0,18,30$

$14,3.75,18,80$

$15,7.5,18,70$

$20,0.0,0.0,1,0$

$30,0.0,0.0,1,1$

$70,0.0,-.000625,0,0$

$80,0.0,0.0,0,0$

$90,0.0,-.00125,0,0$

$100,4000.0,0.20,1 \cdot 0,1 \cdot 0,1 \cdot 0,1.0$

$0.9,1.0,15,1, .000303313$

$4 ., 0, .0001,0.0,0.0$
Explanation

- No. of elements, nodes, analysis code, Guass integration points, no. of load increments, and the magnitude of the load increment.

- Element connectivity input: element no., nodes in that element specified in a counter clockwise direction (max. of 8 nodes per element. Here 4-noded quads are used. For higher order elements, zeros must be replaced by node nos.), material type, and material property number.

- Nodes and the corresponding coordinates: the node number, the $\mathrm{X}$-coordinate, the $Y$-coordinate, and the label for the node based on the boundary condition of the node.
- Node label identification: label number, displacement or force in the X-direct., displacement or force in the Y-direct., 1 if $\mathrm{X}$-displ. is specified 0 if $\mathrm{X}$-force is specified, 1 if $\mathrm{Y}$-displ. is specified 0 if $\mathrm{Y}$-force is specified.

- Properties for a particular element: element property number, Young's modulus, Poisson's ratio, and thicknesses at nodes.

- mode1, mode2, the particular node whose deflection is computed, a switch to solve eigenvalue problems, total mass of the structure. Mode1 and mode 2 are the initial cracking parameters.

$0.10 \mathrm{e}-05, .50, .0066964,0.00112,1.0$ - initial arbitrary and small damage used to get started in the damage subroutine, uniaxial tensile strength, $w$, alpha, mu. The last three are referenced in the text.

- Uniaxial compression strength, switch for hydrostatic compression, epsilon which is used as a measure of tolerance, reference stress and pressure. 
$2,1,1,1$

- ipss.ilest.iwl.ipl. See damage and main for the explanation.

\section{A model shear wall corresponding to}

the input sample of page 31

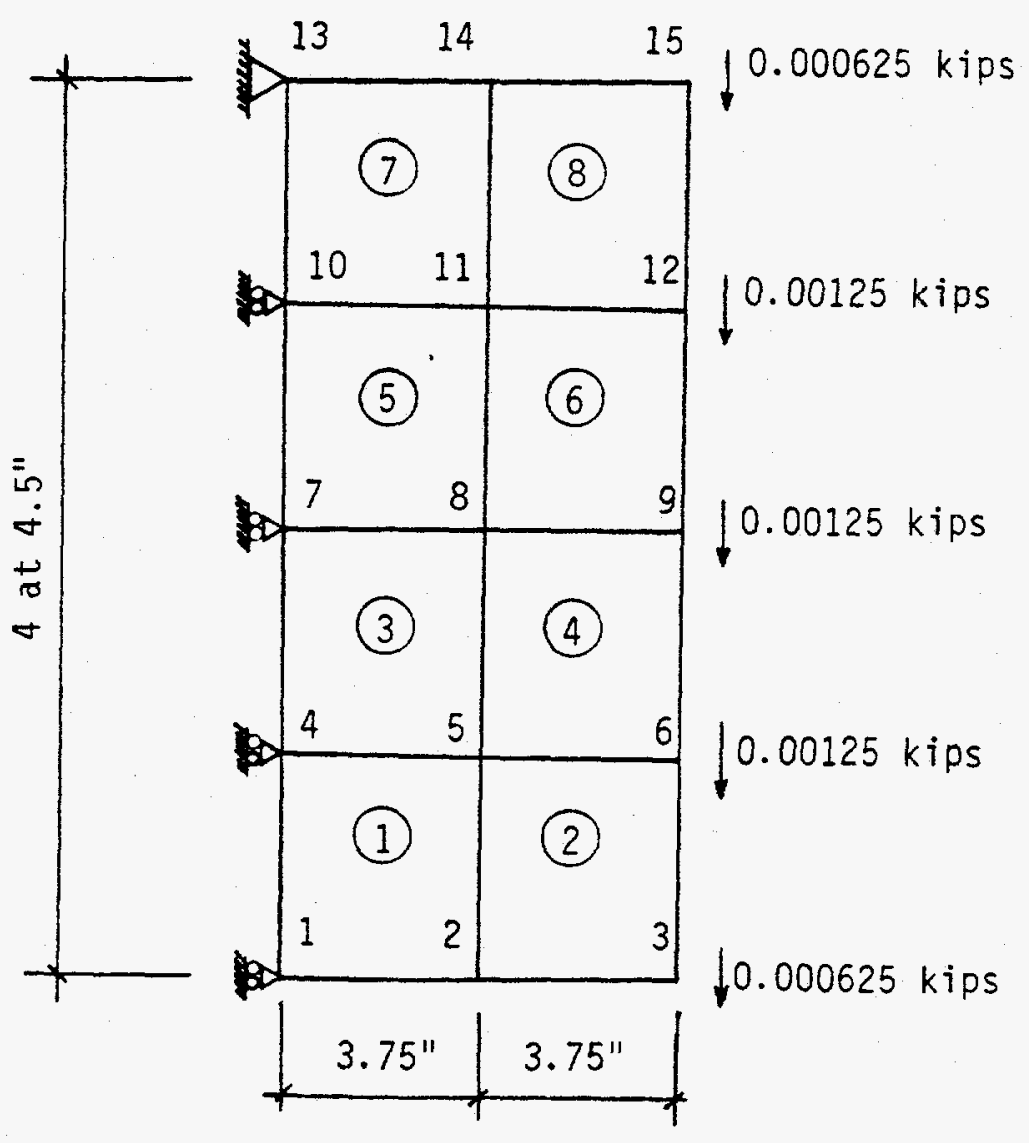




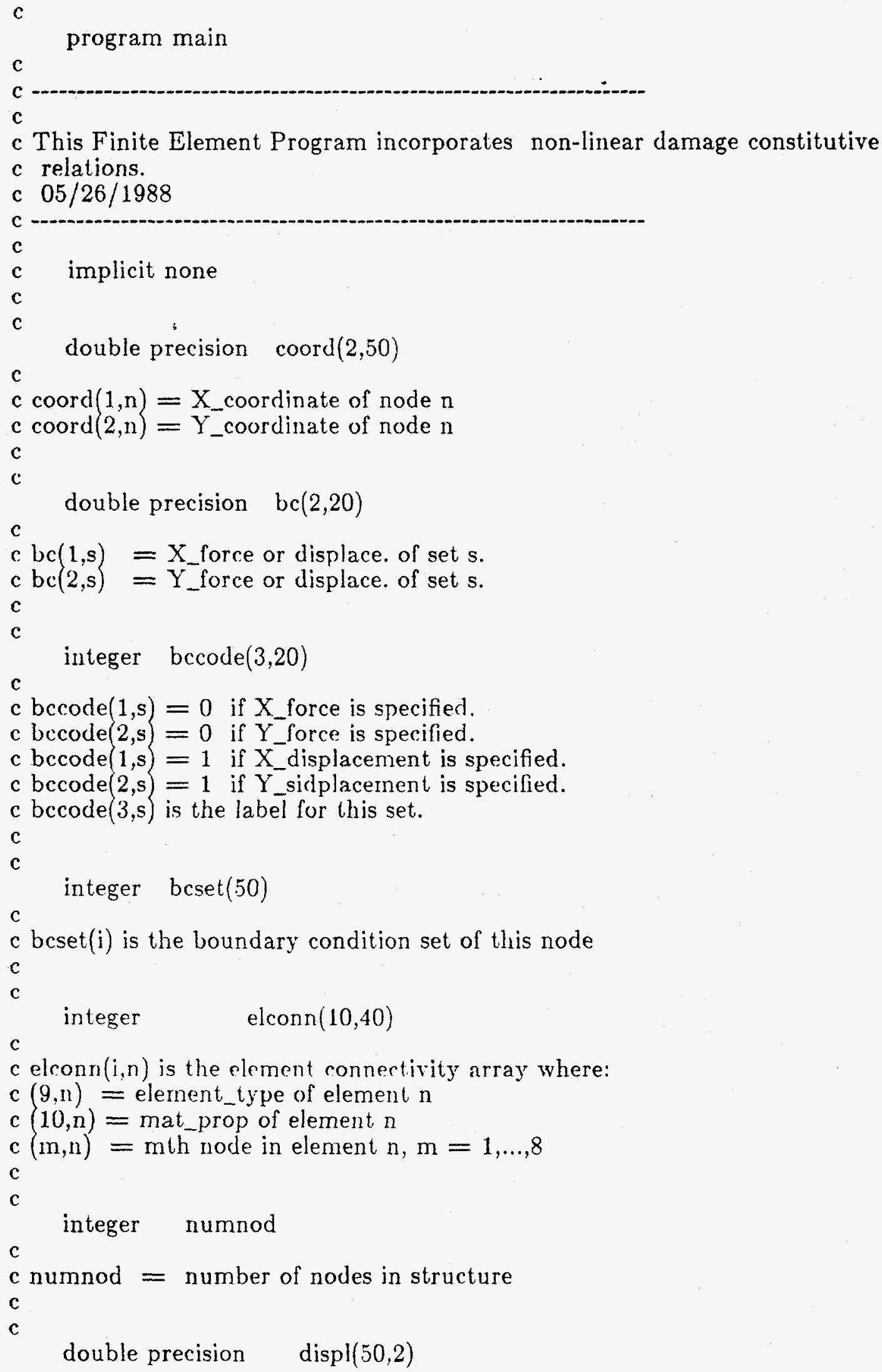


$c$ displ $(50,2)$ is the displacement at nodes.

c

c

integer numelm

c

c numelm is the number of elements in structure.

c

c

integer numeq

c

c numeq is the number of equations to be solved.

c

c

integer code

c

c code ---.-- $1=$ plain stress

c $\quad 2=$ plane strain

c

c

double precision matpro $(7,10)$

c

c matpro $(1,5)=$ label for set

c matpro(2,s) = Young's modulus

c matpro $3, \mathrm{~s})=$ Poisson's ratio

c matpro $4, \mathrm{~s})=$ thickness of material at node 1

c matpro $(5, \mathrm{~s})=$ thichness of material at node 2

c matpro $6, \mathrm{~s})=$ thickness of material at node 3

c matpro $(7, s)=$ thickness of material at node 4

c

double precision $\quad \mathrm{d}(6,6,40,4)$

$c$

c $d(6,6,40,4)$ is the updated elasticity matrix in the

c principal directions.

c

integer eqnum $(2,50)$

c

c eqnum(d,n) is the equation number of node $n$, direction $d$

c $(\mathrm{d}=0,1$ if node is fixed in this direction)

c

c

double precision $\quad A(200,200)$

double precision $\quad \mathrm{B}(200)$

c

c $A(200,200)$ is the $[\mathrm{Kff}]$ in semibanded form.

c $\mathrm{B}(200)$ is the $\{\mathrm{Ff}\}-[\mathrm{Kfs}\}\{\mathrm{ds}\}$ forcing vector.

$\mathrm{c}$

double precision delk,ft,w,alpha,mu,fc,epsl,sgl,pl

c

double precision $\quad \operatorname{disp} 1 \mathrm{t}(50,2)$

double precision

double precision

eigen $1(100)$

double precision

load, $x \mathbf{x}$

deflection

c

c displt(n,i) is the total displacement of node $n$ 
c in the direction of $1=x$-dirertion.

c $\quad 2=y$-direction.

c load is the total force the structure is subjected.after

c $\mathrm{n}$ increment.

$c \mathbf{x x}$ is the total incremental load.

c Deflection is the deflection at the node of interest.

$\mathrm{c}$

integer width

$\mathrm{c}$

c width is the semibandwidth of [Kff].

$c$

c

integer: Gauss

c

c Gauss is the order of integration.

c

c

integer nbcset

integer nmaset

integer nn,iw

integer $\mathrm{ij}, \mathrm{ii}, \mathrm{y}$

integer ipss,itest,iwl,ipl,jp

double precision mode1, mode2, mass

c

c nbcset is the num_bc_set.

c nmaset is the num_mat_sets.

c $\mathrm{ij}$ is the counter.

c ii is a particular node where the calculation

c and plotting of the deflection is desired.

c $\mathrm{y}$ is an integer switch if $\mathrm{y}=1$ then solve eigenvalue problem,

c if $y$ not equal to one, do not sove the

c eigenvalue problem.

c nn is the total number if increment that is specified.

$c$ iwl determines at what interval of the loading increment

c the eigenvalue problem should be solved.

c ipl is the controlling mechanism for plotting. Not used here.

c ipss, itest,jp are explained in the damage subroutine.

c

c

c open input and output files.

c

$$
\begin{aligned}
& \text { open(unit }=1 \text {, file ='input.dat',status='old') } \\
& \text { open(unit }=6, \text { file }=\text { 'output.dat',status='old') } \\
& \text { open(unit }=5, \text { file }=\text { 'pluni',status='old') } \\
& \text { open(unit }=7, \text { file }=\text { 'pleig',status ='old') }
\end{aligned}
$$

c

c input all data.

c

call input(coord, bc, bccode, bcset, elconn, numnod,
! nbcset, nmaset,
! $\quad$ mod,ipss,itest,iwl,ipl,xx numelm, code, matpro, Gauss, nn,delk,ft,w,alpha,mu,fc,jp,epsl,sgl, 
c

c assigen equation numbers to free degrees of freedom.

c

c call aeqnum(bccode, bcset, numnod, eqnum, numeq, nbcset)

c calculate semibandwidth of [Kff].

c call band(elconn, eqnum, numelm, width)

c

do $49 \mathrm{i}=1,50$

do $48 \mathrm{j}=1,2$

$\operatorname{displt}(i, j)=0.0 \mathrm{~d} 0$

48 continue

49 continue

load $=0.0 \mathrm{~d} 0$

write $(5, *)$ displt $(4,1)$, load

$\mathrm{iw}=0$

C

do $100 \mathrm{ij}=1, \mathrm{nn}$

$c$

c nn is the number of load steps taken.

c

$\mathrm{c}$

c calculate element stiffness matrices and write them to stiff.dat.

c call stif(coord, elconn, numelm, matpro, nmaset, code, d, ij)

$\mathrm{c}$

$\mathrm{c}$

$c$ assemble the strusture stiffness matrix $[\mathrm{Kff}]$ and the

c corresponding forcing vector $\{\{\mathrm{Ff}\}-[\mathrm{Kfs}]\{\mathrm{ds}\}\}$

$\mathrm{c}$

call assmbl(elconn, bccode, bcset, bc, eqnum, numeq,

! numelm, numnod, width, A, B,

c

if $(y . e q .1)$ then

$\mathrm{iw}=\mathrm{iw}+1$

if(iw .ne. iwl) go to 21

call principall(A,eigen 1,numeq, mass,numelm)

21 continue

else

continue

endif

c

c Call semibanded equation solver:

c

call solver(numeq, width, $A, B$ )

c

c

c call subroutine to output displacements

c

call disout(coord, bc, bcset, bccode, elconn,

! numnod, B, eqnum, output, numeq, 
c Call subroutine to compute and output strains and stresses.

c

call recovr(elconn, bccode, bcset, bc, eqnum, numeq,

numelin, numnod, coord, output,

code, matpro, displ, nrnaset, $d$,

delk,ft,w,alpha,mu,fc,jp,epsl,sgl, pl,ipss, itest,iwl,ipl,ij,nn,

c mode1, mode2)

c

c

load $=$ load $+x x$

deflection $=\operatorname{dabs}(\operatorname{displt}(\mathrm{ii}, 2))$

write $(5, *)$ deflection, load

if (y.eq. 1) then

if(iw .ne. iwl) go to 22

$\mathrm{iw}=0$

write $(7, *)$ load,dsqrt(dabs(eigen1(numeq)))

22 continue

else

continue

endif

100 continue

c

close (1)

close 6

close $(5)$

$\operatorname{close}(7)$

c

stop

end

c

c

c -

c

subroutine input(coord, bc, bccode, bcset, elconn, numnod,

! numelm, code, matpro, Gauss,

! nbcset, nmaset,

! nn,delk,ft,w, alpha,mu,fc,jp,epsl,sgl,

$! \quad$ pl,ipss,itest,iwl,ipl,xx,

! mode1, mode2, $\mathrm{ii}, \mathrm{y}$, mass)

c

c -

c

c This Subroutine reads the input data to the finite element

c program from a file called 'input.dat'. The information

c read is echoed to an output file called 'output.dat'.

$\mathrm{c}$

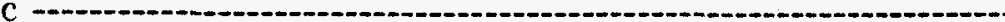

c implicit none

c 
c Variables not described here are explained in the main program.

c double precision coord $(2,50)$

c double precision $b c(2,20)$

c double precision matpro $(7,10)$

$\mathrm{c}$ double precision delk,ft,w,alpha, $\mathrm{mu}, f \mathrm{c}, \mathrm{epsl}, \mathrm{sgl}, \mathrm{pl}, \mathrm{xx}$

c double precision mode1, mode2, mass

c integer bccode $(3,20)$

c integer bcset $(50)$ integer elconn $(10,40)$

c integer numnod

c integer numelm

c integer code

c integer Gauss

c integer $\mathrm{nn}, \mathrm{ipss}, \mathrm{itest,iwl,ipl,jp}$

c

character*72 title

integer $\mathrm{i}, \mathrm{ii}, \mathrm{y}$

integer $\mathbf{j}$

in eger nbcset

integer nmaset

integer node

integer elem

integer flag

c

c node is the node number.

$c$ elem is the element number.

c

c -

c

c read and write the tille of the problem

c

$\operatorname{read}(1, *)$ title

write $(6,1011)$

write 6,1000$)$

write $\left(6,{ }^{*}\right)$ title

c

c read and write some problem parameters.

c

$\operatorname{read}\left(1,{ }^{*}\right)$ numnod, numeln, code, Gauss, nn, xx

write $(6,1011)$

c

write $(6,1020)$ numnod, numelm, code, Gauss 
c read and write element connectivity, type, and material number.

c

do $10 \mathrm{i}=1$, numelm

$\operatorname{read}(1, *)$ elem, $(\operatorname{elconn}(\mathrm{j}$, elem $), \mathrm{j}=1,10)$

10 continue

write $(6,1011)$

write 6,1025$)$

c

write $(6,1010)$

do $15 \mathrm{i}=1$, numelm

write $(6,1030) \mathrm{i},(\operatorname{elconn}(\mathrm{j}, \mathrm{i}), \mathrm{j}=1,10)$

15 continue

c

c determine how many material property sets there are:

$\mathrm{c}$

nmaset $=0$

do 25 elem $=1$, numelm

flag $=0$

do $20 \mathrm{i}=1$, elem - 1

if (elcoun $(10$, elem $)$.eq. elconn $(10, i))$ then

flag $=1$

else

continue

endif

20 continue

$\mathrm{if}(\mathrm{flag}$.eq. 0$)$ nmaset $=\mathrm{n}$ maset +1

25 continue

c

c read and write nodal coordinates and boundary condition sets.

c

do $30 \mathrm{i}=1$, numnod

$\operatorname{read}(1, *)$ node, $($ coord $(j$, node $), j=1,2), \operatorname{bcset}($ node $)$

30 continue

c

write $(6,1011)$

write $(6,1029)$

write $(6,1010)$

do $35 \mathrm{i}=1$, numnod

write $(6,1040) \mathrm{i},(\operatorname{coord}(\mathrm{j}, \mathrm{i}), \mathrm{j}=1,2), \operatorname{bcset}(\mathrm{i})$

35 continue

c

c determine how many boundary condition sets there are:

c

nbcset $=0$

do 45 node $=1$, numnod

flag $=0$

do $40 \mathrm{i}=1$, node -1

if(bcset(node) .eq. bcset(i)) then

flag $=1$

else

continue

endif

40 continue

if(flag .eq. 0$)$ nbcset $=$ nbcset +1 


\section{5 continue}

c

$c$ read and write none zero applied force or displacement

c boundary conditions.

c

$$
\text { do } 50 \mathrm{i}=1 \text {, nbcset }
$$

$\operatorname{read}(1, *) \operatorname{bccode}(3, \mathrm{i}),(\mathrm{bc}(\mathrm{j}, \mathrm{i}), \mathrm{j}=1,2),(\operatorname{bccode}(\mathrm{j}, \mathrm{i})$,

50 continue

$$
j=1,2)
$$

c

write $(6,1011)$

write $(6,1045)$

write $6 ; 1010)$

write $(6,1036)$

write $(6,1038)$

write $(6,1039)$

write $(6,1010)$

do $55 \mathrm{i}=1$, nbcset

write $(6,1050)$ bccode $(3, I),(b c(j, i), j=1,2),(\operatorname{bccode}(j, i)$, !

55 continue $\mathrm{j}=1,2$ )

c

c read and write material properties for each material type.

c

do $60 \mathrm{i}=1$, nmaset

$\operatorname{read}(1, *)(\operatorname{matpro}(\mathrm{j}, \mathrm{i}), \mathrm{j}=1,7)$

write $(6, *)$ matpro $(1,1)$, matpro $(2,1)$, matpro(3,1), matpro $(4,1)$

60 continue

c

write $(6,1011)$

write $(6,1053)$

write 6,1010$)$

write $(6,1055)$

write $(6,1010)$

do $65 \mathrm{i}=1$,nmaset

write $(6,1060)$ idint(matpro $(1, i)),(\operatorname{matpro}(j, i), j=2,4)$

65 continue

c

write $(6,1011)$

write $(6,1070)$

write $(6,1010)$

c

$\operatorname{read}(1, *)$ mode 1, mode $2, \mathrm{ii}, \mathrm{y}$, mass

read $(1, *)$ delk, ft,w,alpha.mu

read $1, *\}$ fc,jp,epsl,sgl,pl

$\operatorname{read}(1, *)$ ipss, itest, iwl,ipl

return

c

1000 format'

1010 format $(/)$

OUTPUT OF PROGRAM MAIN '/)

1011 format $/ /$ )

1020 format ('NUMBER OH NODAL POINTS $=$ ',15/

$!$ 'NUMBER OF ELEMEN'TS

! ANALYSIS CODE

$=, \overline{15 /}$ 


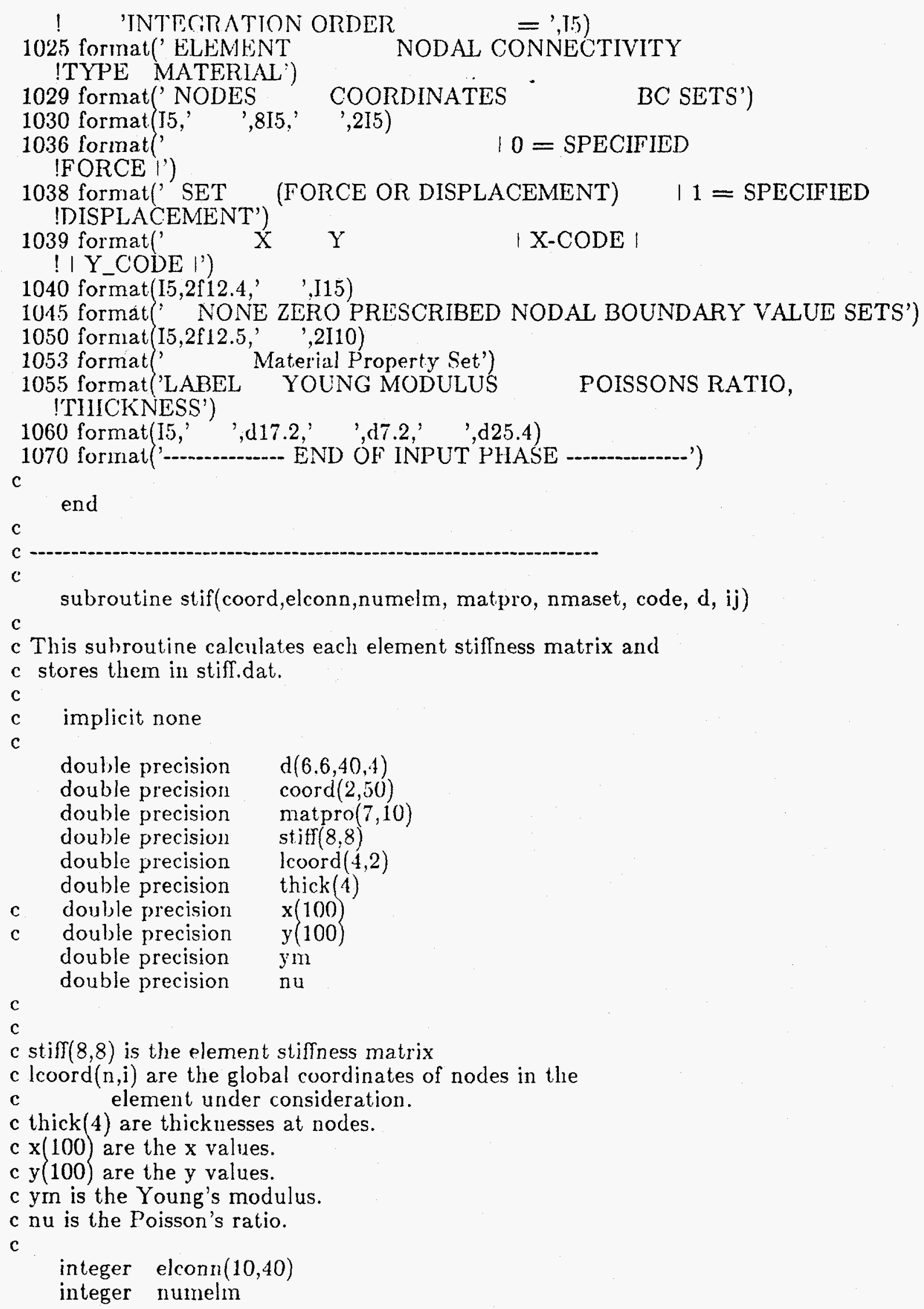




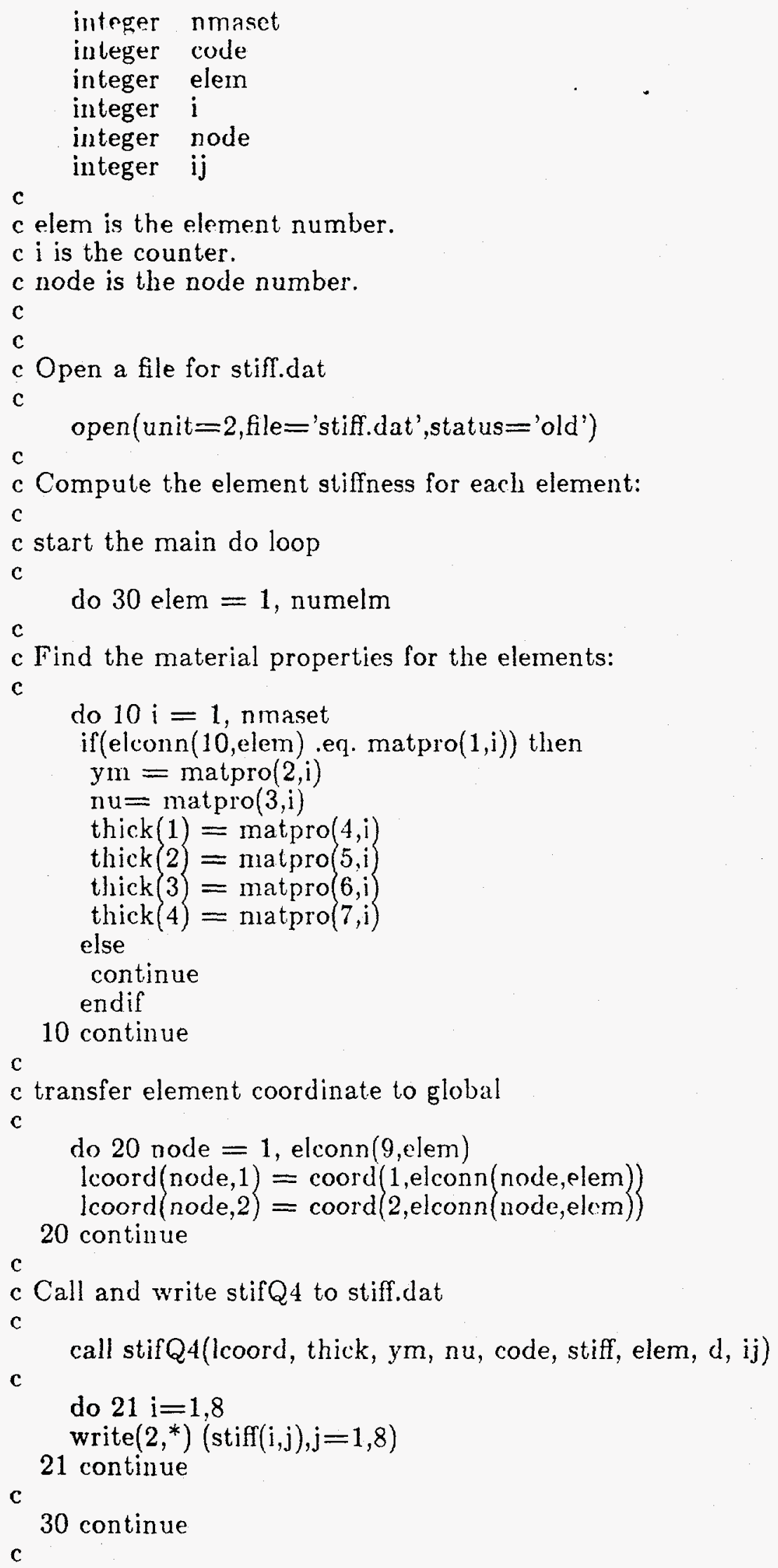




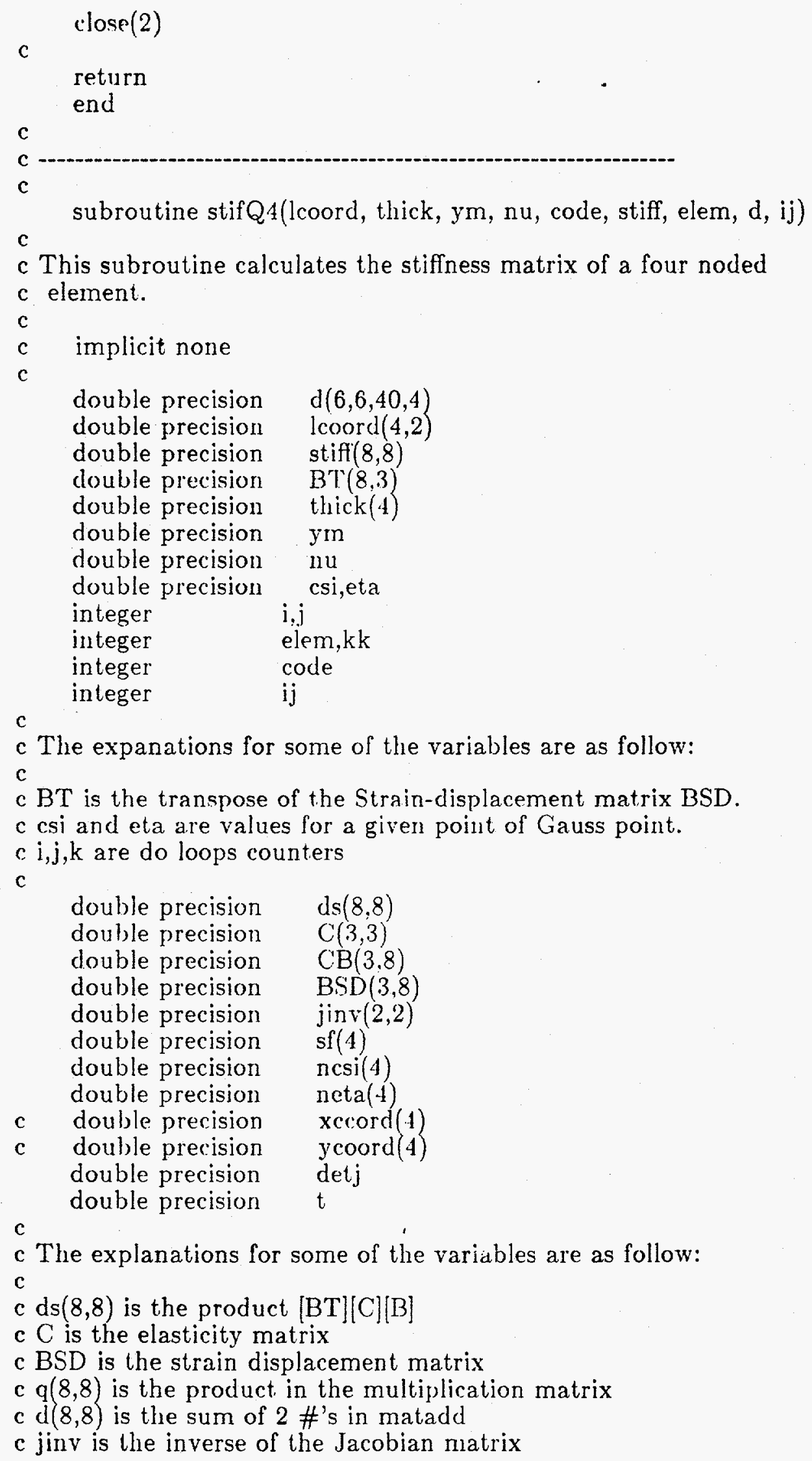


c sf(1) arr the shape functions at nodes

c ncsi(4) are the partial of n wrt csi

c neta(4) are the partial of $n$ wrt eta

$c$ xcoord are the $x$ coordinates of nodes

c ycoord are the $y$ coordinates of nodes

c detj is determinant of Jacobian

$c t$ is the thickness tinies sf.

c

C

c Initialize stiff(i,j)

c

do $20 \mathrm{i}=1,8$

do $10 \mathrm{j}^{\mathrm{t}}=1,8$

$\operatorname{stiff}(i, j)=0.0$

10 conlinue

20 continue

c

c

c Call the elsticity matrix $\mathrm{C}$

c

c

c

do $90 \mathrm{kk}=1,4$

call elast. (ym, nu, code, C, ij, d, elem, kk)

c

c Use 2 Gaussian points

c Evaluate everything at 4 points

c For $n=2$, csi and ela are both .57735

c Construct a do loop for each point

c

call nn4(csi,eta,kk)

call $\mathrm{n} 4$ (sf,ncsi,neta,csi,eta)

call jacob(lcoord,ncsi,neta,jinv,detj)

$\mathrm{c}$

c Calculate the thickness

c

$$
\begin{aligned}
& \mathrm{t}=0.0 \\
& \text { do } 40 \mathrm{i}=1,4 \\
& \mathrm{t}=\mathrm{t}+\mathrm{s} f(\mathrm{i})^{*} \text { thick }(\mathrm{i})
\end{aligned}
$$

40 continue

c

$$
\text { call stdisp(RSD.nesi.net n.jinv.sf,code) }
$$

c

c Calcluate $B$ transpose

c

$$
\begin{aligned}
& \text { do } 60 \mathrm{i}=1,8 \\
& \text { do } 50 \mathrm{j}=1,3
\end{aligned}
$$

50 continue

60 continue

c

c Multiply BT times C times B

c

call mult(C,BSD, $3,3,8, \mathrm{CB})$ 


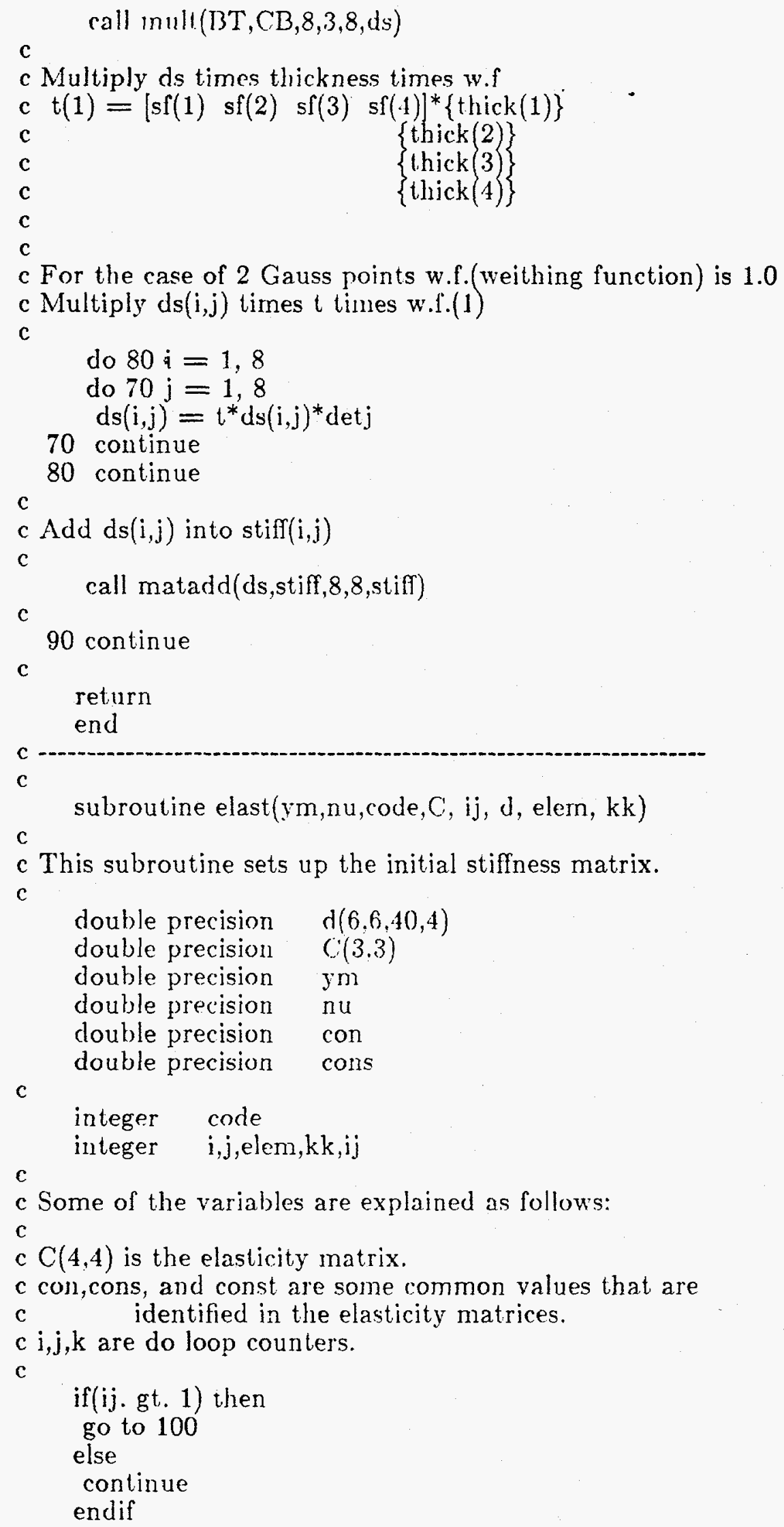


$c$

c Initialize Elasticity Matrix:

c

do $20 \mathrm{i}=1,3$

do $10 \mathrm{j}=1,3$

$\mathrm{C}(\mathrm{i}, \mathrm{j})=0.0 \mathrm{~d} 0$

10 continue

20 continue

c

c

c Define the elasticity matrix for the plain strain problems:

c

if(code seq. 2) then

con $=\mathrm{ym} /\left(1.0 \mathrm{~d} 0-\mathrm{nu}^{* * 2.0 \mathrm{~d} 0)}\right.$

$\mathrm{C}(1,1)=\mathrm{con}$

$\mathrm{C}(1,2)=\mathrm{con}^{*} \mathrm{nu}$

$\mathrm{C}(2,1)=\mathrm{C}(1,2)$

$\mathrm{C}(2,2)=\mathrm{con}$

$\mathrm{C}(3,3)=\operatorname{con}^{*}(1.0 \mathrm{~d} 0-\mathrm{nu}) / 2.0 \mathrm{~d} 0$

else

continue

endif

C

c Define the elasticity matrix for the plain stress problem:

c

if (corle .eq. 1) then

cons $=\left(\mathrm{ym}^{*}(1.0 \mathrm{~d} 0-\mathrm{nu})\right) /\left((1.0 \mathrm{~d} 0+\mathrm{nu})^{*}(1.0 \mathrm{~d} 0-2.0 \mathrm{~d} 0 * \mathrm{nu})\right)$

$\mathrm{C}(1,1)=\mathrm{cons}$

$\mathrm{C}(1,2)=$ cons ${ }^{*} \mathrm{u} /(1.0 \mathrm{~d} 0-\mathrm{nu})$

$C(2,1)=C(1,2)$

$\mathrm{C}(2,2)=$ cons

$C(3,3)=$ cons $^{*}(1.0 \mathrm{~d} 0-2.0 \mathrm{~d} 0 * n u) /(2.0 \mathrm{~d} 0 *(1.0 \mathrm{~d} 0-\mathrm{nu}))$

else

continue

endif

go to 200

c

100 continue

c

$\mathrm{C}(1,1)=\mathrm{d}(1,1$, elem, $\mathrm{kk})$

$C(1,2)=d(1,2$, elem, kk $)$

$C(2,1)=C(1,2)$

$\mathrm{C}(2,2)=\mathrm{d}(2,2$, elem,kk $)$

c

200 continue

c

return

end

c

c

subroutine $\mathrm{n} 4$ (sf,ncsi,neta,csi,eta)

c

c This subroutine calculates the shape functions and the derivatives. 
C

double precision double precision double precision double precision

$$
\begin{aligned}
& \operatorname{sf}(4) \\
& \operatorname{ncsi}(d) \\
& \text { neta(d) } \\
& \operatorname{csi} \\
& \text { eta } \\
& a, b, c, d
\end{aligned}
$$$$
\text { double precision }
$$

c sf(4) designate values of the shape function at each point.

c ncsi(4) are the partial derivative of shape functions W.R.T. csi.

c neta(4) are the partial derivative of shape functions W.R.T. eta.

$c$ csi and eta are the local coordinates of the elements.

$c a, b, c$, and $d$ are for the ease of calculations.

c

c Define a, b, c, and d:

c

$$
\begin{aligned}
& a=(1.0 \mathrm{~d} 0+\mathrm{csi}) \\
& b=(1.0 \mathrm{~d} 0-\mathrm{csi}) \\
& c=(1.0 \mathrm{~d} 0+\text { eta }) \\
& \mathrm{d}=(1.0 \mathrm{~d} 0-\text { eta })
\end{aligned}
$$

c

$$
\begin{aligned}
& \operatorname{sf}(1)=.25 \mathrm{~d} 0 * \mathrm{~b} * \mathrm{~d} \\
& \operatorname{sf}(2)=.25 \mathrm{~d} 0 * \mathrm{a} * \mathrm{~d} \\
& \operatorname{sf}(3)=.25 \mathrm{~d} 0 * \mathrm{a} * \mathrm{c} \\
& \operatorname{sf}(4)=.25 \mathrm{~d} 0 * \mathrm{~b} * \mathrm{c}
\end{aligned}
$$

c

c Calculate the Partials of Shape Functions W.R.T. csi:

$$
\text { c }
$$

$$
\begin{aligned}
& n \operatorname{csi}(1)=-.25 \mathrm{~d} 0 * \mathrm{~d} \\
& n \operatorname{csi}(2)=+.25 \mathrm{~d} 0 * \mathrm{~d} \\
& \operatorname{ncsi}(3)=+.25 \mathrm{~d} 0 * \mathrm{c} \\
& \mathrm{n} \operatorname{csi}(4)=-.25 \mathrm{~d} 0 * \mathrm{c}
\end{aligned}
$$

c

c Calculate the Partials of the Shape Functions W.R.T. eta:

$\mathrm{c}$

$$
\begin{aligned}
& \operatorname{neta}(1)=-.25 \mathrm{~d} 0 * \mathrm{~b} \\
& \text { neta }(2)=-.25 \mathrm{~d} 0 * \mathrm{a} \\
& \operatorname{neta}(3)=+.25 \mathrm{~d} 0 * \mathrm{a} \\
& \operatorname{neta}(4)=+.25 \mathrm{~d} 0 * \mathrm{~b}
\end{aligned}
$$

C

return

end

c

$\mathrm{c}$

C

$\mathrm{c} 234567$

c

c

subroutine nn4(csi,eta,kk)

c This subroutine specifies local coordinates.

c

double precision csi

double precision eta

integer $\mathrm{kk}$ 
c

if(kk.eq.1) then

csi $=-.57735$

eta $=$ csi

else

continue

endif

if (kk.eq. 2$)$ then

$\mathrm{csi}=.57735$

eta $=-.57735$

else

continue

endif :

c

if(kk.eq.3) then

$\mathrm{csi}=.57735$

eta $=\mathrm{csi}$

else

continue

endif

c

if $(k k . e q .4)$ then

$\mathrm{csi}=-.57735$

eta $=+.57735$

else

continue

endif

c

return

end

c

c

c

c234567

subroutine jacob(lcoord,ncsi,neta,jinv,detj)

c

c This subroutine forms the Jacobian of transformation and

c its inverse.

c

double precision lcoord $(4,2)$

double precision $\mathrm{j}(2,2)$

double precision jinv $(2,2)$

double precision

double precision

double precision

$\operatorname{ncsi}(4)$

neta $(4)$

c

integer $\mathrm{i}, \mathrm{k}$

c

c $j(2,2)$ is the Jocobian matrix.

c jinv $(2,2)$ is the inverse of the Jacobian.

$c$ det $j$ is the determinant of the Jacobian matrix.

$\mathrm{c} n$ is the number of nodes.

c

c Initialize the Jacobian and its inverse Matrices: 
c

do $20 \mathrm{i}=1,2$

do $10 \mathrm{k}=1,2$

$\mathrm{j}(\mathrm{i}, \mathrm{k})=0.0 \mathrm{~d} 0$

jinv $(\mathrm{i}, \mathrm{k})=0.0 \mathrm{~d} 0$

10 continue

20 continue

c

c Multiply the matrix of shape function, [sf], by the coordinates

c of the nodes.

c

$$
\begin{aligned}
& \text { do } 30 \mathrm{i}=1,4 \\
& \mathrm{j}(1,1) \pm \mathrm{j}(1,1)+\operatorname{ncsi}(\mathrm{i}) * \operatorname{lcoord}(\mathrm{i}, 1)
\end{aligned}
$$$$
\mathrm{j}(1,2)=\mathrm{j}(1,2)+\operatorname{ncsi}(\mathrm{i}) * \operatorname{looord}(\mathrm{i}, 2)
$$$$
\mathrm{j}(2,1)=\mathrm{j}(2,1)+\operatorname{neta}(\mathrm{i}) * \operatorname{lcoord}(\mathrm{i}, 1)
$$$$
\mathrm{j}(2,2)=\mathrm{j}(2,2)+\operatorname{neta}(\mathrm{i}) * \operatorname{Icoord}(\mathrm{i}, 2)
$$

30 continue

c

c Calculate the determinant of the Jacobian:

$\mathrm{c}$

$$
\operatorname{det} j=j(1,1)^{*} \mathrm{j}(2,2)-\mathrm{j}(2,1)^{*} \mathrm{j}(1,2)
$$

$\mathrm{c}$

c Calculate the inverse of $\mathrm{j}(2,2)$ :

$\mathrm{c}$

$$
\begin{aligned}
& \operatorname{jinv}(1,1)=\mathrm{j}(2,2) / \text { detj } \\
& \operatorname{jinv}(1,2)=-\mathrm{j}(1,2) / \text { det } \mathrm{j} \\
& \operatorname{jinv}(2,1)=-\mathrm{j}(2,1) / \text { detj } \\
& \operatorname{jinv}(2,2)=\mathrm{j}(1,1) / \text { detj }
\end{aligned}
$$

c

c

return

end

c

c

c

c234567

subroutine stdisp(BSD, ncsi, neta,jinv,sf,code)

c

c Strain-displacement relations are formed in this subroutine.

c

double precision

double precision

double precision

double precision

double precision

double precision

$\operatorname{BSD}(3,8)$

jinv $(2,2)$

nesi(4)

neta $(4)$

$\mathrm{sf}(4)$

double precision

$\mathrm{q}(4)$

$\mathrm{p}(4)$

c

integer code

integer $i, 1$

c

c $\operatorname{BSD}(3,8)$ is the Strain-Displacement Matrix.

c $q(4)$ is the partial of [sf] w.r.t. $x$.

c $\mathrm{p}(4)$ is the partial of [sf] w.r.t. $y$ 
c

c

c Initialize the B matrix:

c

do $20 \mathrm{i}=1,3$

do $10 l=1,8$

$\operatorname{BSD}(\mathrm{i}, \mathrm{l})=0.0 \mathrm{~d} 0$

10 continue

20 continue

c

do $25 \mathrm{i}=1,4$

$\mathrm{q}(\mathrm{i})=0.0 \mathrm{~d} 0$

$\mathrm{p}(\mathrm{i})=0.0 \mathrm{~d} 0$

25 continue

c

c Generate the B matrix:

c

$$
\begin{aligned}
& \text { do } 30 \mathrm{i}=1,4 \\
& \mathrm{q}(\mathrm{i})=\operatorname{ncsi}(\mathrm{i})^{*} \operatorname{jinv}(1,1)+\operatorname{neta}(\mathrm{i})^{*} \operatorname{jinv}(1,2)
\end{aligned}
$$

$\mathrm{p}(\mathrm{i})=\operatorname{ncsi}(\mathrm{i}) * \mathrm{jinv}(2,1)+\operatorname{neta}(\mathrm{i}) * \mathrm{jinv}(2,2)$

30 continue

c

$\operatorname{BSD}(1,1)=q(1)$

$\mathrm{BSD}(1,3)=\mathrm{q}(2)$

$\mathrm{BSD}(1,5)=\mathrm{q}(3)$

$\operatorname{BSD}(1,7)=\mathrm{q}(4)$

$\operatorname{BSD}(2,2)=p(1)$

$\operatorname{BSD}(2,4)=p(2)$

$\operatorname{BSD}(2,6)=\mathrm{p}(3)$

$\operatorname{BSD}(2,8)=\mathrm{p}(4)$

$\operatorname{BSD}(3,1)=\mathrm{p}(1)$

$\operatorname{BSD}(3,2)=\mathrm{q}(1)$

$\operatorname{BSD}(3,3)=p(2)$

$\mathrm{BSD}(3,4)=\mathrm{q}(2)$

$\mathrm{BSD}(3,5)=\mathrm{p}(.3)$

$\operatorname{BSD}(3,6)=\mathrm{q}(3)$

$\operatorname{BSD}(3,7)=p(4)$

c

$$
\operatorname{BSD}(3,8)=q(4)
$$

c

return

end

c

c

c23456

subroutine mult (A,B,NRA,NCA,NCB,CB)

c

c This is a multiplication algorithm for matrices.

$\mathrm{c}$
integer
integer
integer
integer
NRA
NCA
$\mathrm{NCB}$
$\mathrm{i}, \mathrm{j}, \mathrm{k}$ 
c

double precision $\quad \mathrm{A}(\mathrm{NRA}, \mathrm{NCA})$

double precision $B(N C A, N C B)$

c

double precision

$\mathrm{CB}(\mathrm{NRA}, \mathrm{NCB})$

c $C B(N R A, N C B)$ is the product of $[A]$ and $[B]$.

c NRA is the number of rows is $A$.

c NCA is the number of Columns in $A$.

c NCB is the number of columins in $B$

$c \mathrm{i}, \mathrm{j}, \mathrm{k}$ are the do loop variables.

c

c Initialize the $[\mathrm{CB}]$ matrix:

$c$

do $20 \mathrm{j}=1$, NRA

do $10 \mathrm{j}=1, \mathrm{NCB}$

$\mathrm{CB}(\mathrm{i}, \mathrm{j})=0.0 \mathrm{do}$

10 continue

20 continue

c

c Multiply $[A]$ times $[B]$ :

c

do $50 \mathrm{i}=1$, NR $\Lambda$

do $40 \mathrm{j}=1, \mathrm{NCB}$

do $30 \mathrm{k}=1, \mathrm{NCA}$

$\mathrm{CB}(\mathrm{i}, \mathrm{j})=\mathrm{CB}(\mathrm{i}, \mathrm{j})+\mathrm{A}(\mathrm{i}, \mathrm{k}) * \mathrm{~B}(\mathrm{k}, \mathrm{j})$

30 continue

40 continue

50 continue

c

return

end

c

c -

c

c234567

subroutine matadd(A,B,NRA,NCA,D)

c

c This is an algorithm for addition of matrices.

integer NRA

integer NCA

integer $\mathrm{i}, \mathrm{j}$

$c$

double precision

double precision

$A(N R A, N C A)$

B(NRA,NCA)

double precision

D(NRA,NCA)

$\mathrm{c}$

c NRA is the number of rows in the matrix $A$.

c NCA id the number of cloumns in the matrix $A$.

c $\mathrm{i}, \mathrm{j}$ are the variables for do-loops.

c

c Add A to B:

c

do $20 \mathrm{i}=1, \mathrm{NRA}$ 


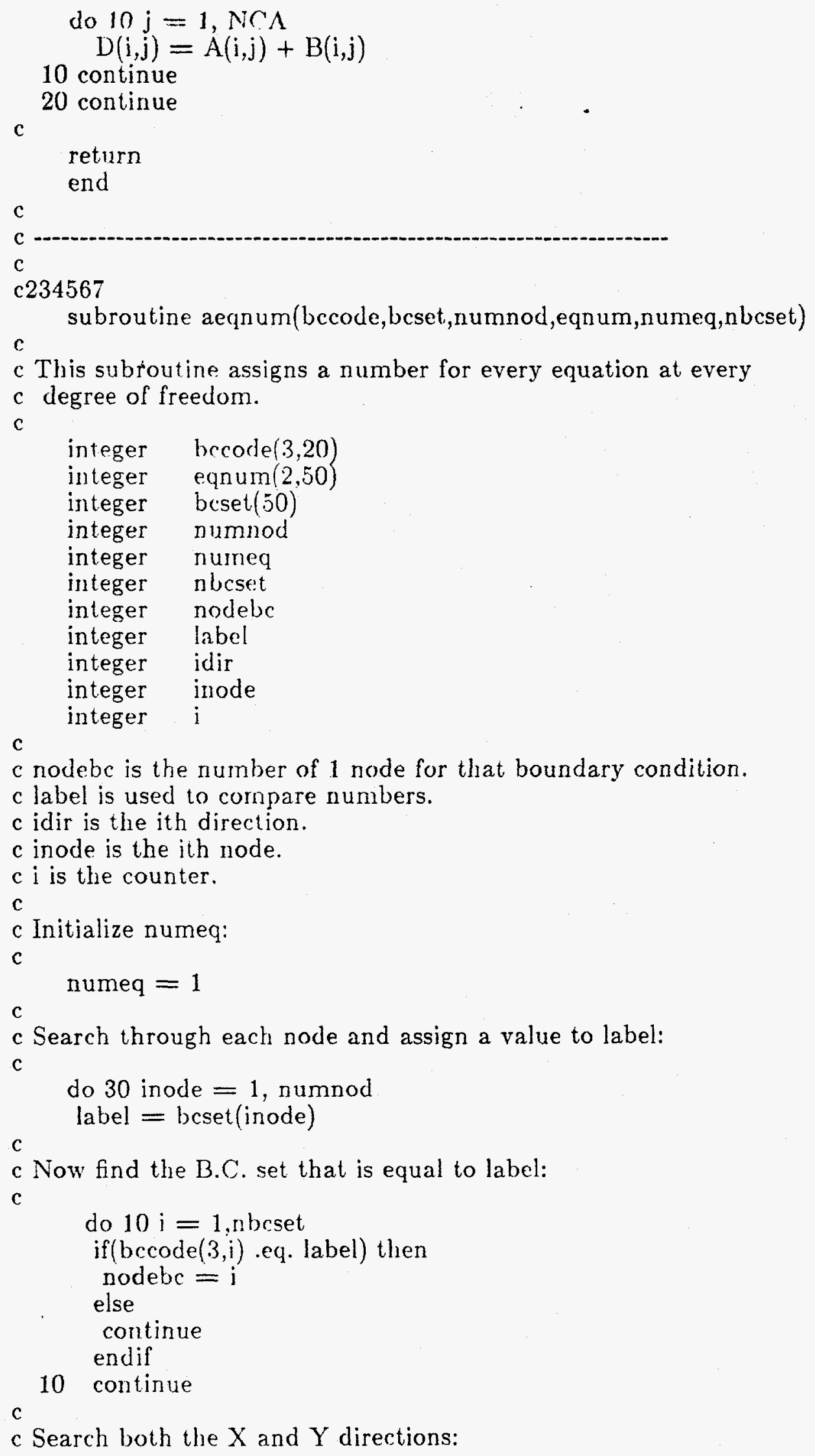

$c$ Search both the $\mathrm{X}$ and $\mathrm{Y}$ directions: 
$\mathrm{c}$

do $20 \mathrm{idir}=1,2$

if(bccode(idir,nodebc) .eq. 0) then

eqnum(idir,inode) $=$ numeq

numeq $=$ numeq +1

else

eqnum(idir, inode $)=0$

endif

20 continue

30 continue

c234567

c

numeq $=$ numeq -1

return

end

c

c

c

c234567

subroutine band(elconn, eqnum, numelm, width)

c

c This subroutine finds the semi-bandwith of [Kff].

c

integer elconn $(10,40)$

integer eqnum $(2,50)$

integer numelin

integer width

integer eqelma

integer eyelmi

integer elindif

integer maeldi

integer nelnod

integer elem

integer idir

c

integer inode

c eqelma is the element's maximum equation number.

c egelini is the elemnet's minimum equation number.

c elmdif is the difference between 2 nodes.

c maeldi is the maximum differnece of two equations in an element.

$c$ nelnod is the number of element nodes.

$\mathrm{c}$

c Initialize variables to zero:

c

elmdif $=0$

maeldi $=0$

eqelma $=0$

c

c Make eqelmi (EQ_ELEM_MIN) larger than your greatest number of

c equations(2000).

c

c Search each element:

c

do 30 elem $=1$, numelm 


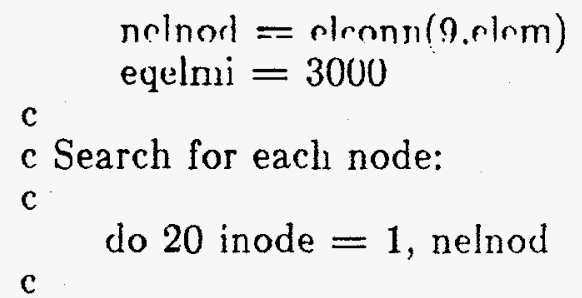

c Search in the $\mathrm{X}$ and $\mathrm{Y}$ directions and find the maximum and

c minimum element equations:

c do $10 \mathrm{idir}=1,2$ eqelma $=\max ($ eqnum(idir,elconn(inode,elem) $)$,eqelma $)$ eqelmi $=\min ($ eqnum (idir,elcomn(inode,elenı)), eqelmi) 10 contihue

c

20 continue

$\mathrm{c}$

c Find the maximum difference:

c elmdif = eqelma - eqelni

c

c Find the maximum element difference:

c

maeldi $=\max ($ maeldi,elmdif $)$

c

30 continue

c

c Find the semi_bandwidth:

c

width $=$ maeldi +1

c

return

end

c

c

c

c234567

subroutine assmbl(elconn,bccode,bcset,bc,eqnum,numeq, * numelm,numnod, width, $A, B$, nbcset)

C

c This subroutine assembles the stiffness matrix.

c

c

$\begin{array}{ll}\text { integer } & \text { elconn }(10,40) \\ \text { integer } & \text { bccode }(3,20) \\ \text { integer } & \text { eqnum(2,50) } \\ \text { integer } & \text { bcset }(50) \\ \text { integer } & \text { numnod } \\ \text { integer } & \text { numelm } \\ \text { integer } & \text { numer } \\ \text { integer } & \text { width } \\ \text { integer } & \text { node,elem,n1,n2 } \\ \text { integer } & \text { node1,node2,dir,dr } \\ \text { integer } & \text { row,col,i }\end{array}$


integer brflag
integer bcnum
integer nbcset
c double precision

double precision

double precision

double precision

double precision

double precision

\section{$\mathrm{A}(200,200)$}

$\mathrm{B}(200)$

stiff $(8,8)$

bc $(2,20)$

spdisp

spforc

c

c $A$ is the stiffness matrix Kfr.

$c B$ is the forcing vector.

c Stiff is the stiffness matrix.

c spdisp is the specified displacement.

c spforc is the specified force.

c

c Open file:

c

$$
\text { open(unit }=2 \text {,file }=\text { 'stifl.dat',status }=\text { 'old') }
$$

c

c Initialize the matrix A:

c

do $1 \mathrm{i}=1$,numeq

do $1 \mathrm{j}=1$, numeq

$A(i, j)=0.0 \mathrm{~d} 00$

$\mathrm{B}(\mathrm{i})=0.0 \mathrm{~d} 00$

1 continue

c

row $=0$

$\mathrm{col}=0$

c diag

do 60 elem $=1$, numelm

do $5 \mathrm{i}=1,8$

5 $\operatorname{read}\left(2,{ }^{*}\right)(\operatorname{stiff}(i, j), j=1,8)$

continue

do $50 \mathrm{n} 1=1$,elconn $(9$,elem)

node $1=\operatorname{elconn}(\mathrm{n} 1$,elem $)$

c write $\left(6,{ }^{*}\right)$ 'we are in subroutine assemble line \#1224'

c write $\left(6,{ }^{*}\right) ' \mathrm{ij}={ }^{\prime}, \mathrm{ij},{ }^{\prime} \mathrm{kk}={ }^{\prime}, \mathrm{kk}$

do 40 dir $=1,2$

row $=$ eqnuns (dir.nodr: 1$)$

do $30 \mathrm{n} 2=1$, elconn $(9$,elem $)$

node $2=\operatorname{elconn}(\mathrm{n} 2$,elem $)$

do $20 \mathrm{dr}=1,2$

$\mathrm{col}=$ eqnum $(\mathrm{dr}$, node 2$)$

if(row.gt.0) then

if(col.gt.0) then

if $($ col-row +1$) \cdot g t .0)$ then

* $\mathrm{A}($ row,$($ col-row +1$))=\mathrm{A}($ row,$($ col-row +1$))+\operatorname{stiff}(((\mathrm{n} 1-1)$

else $\left.\left.*^{2}+\mathrm{dir}\right),\left((\mathrm{n} 2-1)^{*} 2+\mathrm{dr}\right)\right)$

continue

endif 


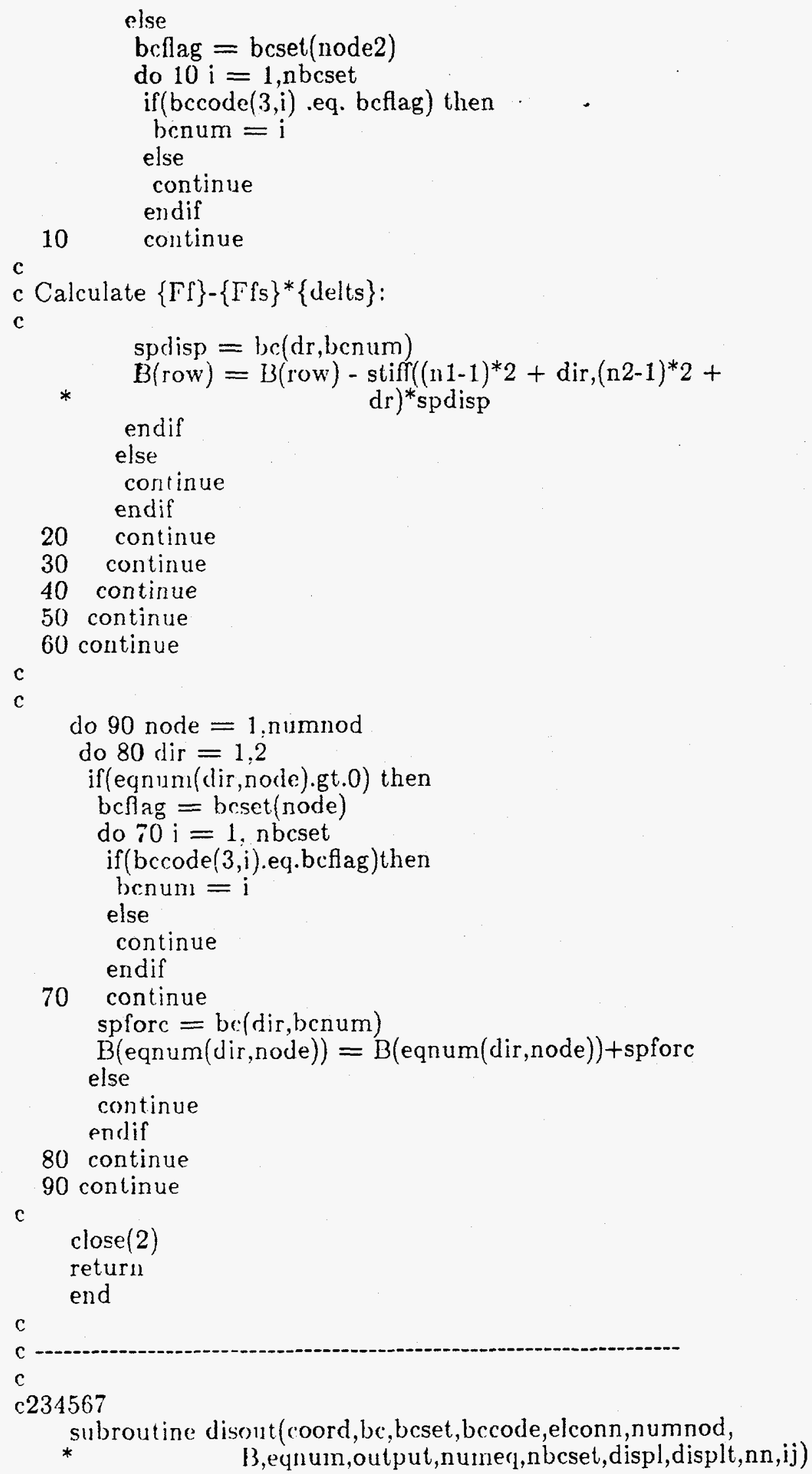


C

c This subroutine calculates the incremental displacements.

c

integer elconn $(10,40)$

integer eqnum $(2,50)$

integer bccode $(3,20)$

integer $b \operatorname{cset}(50)$

integer numey

integer nummod

integer output

integer idir,inode, $\mathrm{i}$

integer label,check

integer nbcset

c

integer $\mathrm{nn}, \mathrm{ij}$

double precision $\operatorname{coord}(2,50)$

double precision $\quad b c(2,20)$

double precision displ(50,2)

double precision displt $(50,2)$

double precision $\quad B($ numeq)

double precision $\quad \mathrm{D}(2)$

c

c displ is the nodal displacement matrix.

c $\mathrm{B}$ is the displacement vector.

c D is a local variable for writing the displacements.

c output is the output number.

c label and check are progrannming flags.

c

c

if(ij.eq. nn)then

write $(6,177)$

177 format.' G_NODE',2x,'X DISP',6x,'Y DISP')

else

continue

endif

c

do 30 inode $=1$, numnod

do 20 idir $=1,2$

if (eqnum(idir, inode).eq.0) then

label $=$ beset(inode)

do $10 \mathrm{i}=1$, nbcset

if (label.eq.bccode( $3, \mathrm{i}))$ then

check $=\mathrm{i}$

$\mathrm{D}(\mathrm{idir})=\mathrm{bc}(\mathrm{idir}, \mathrm{check})$

else

continue

endif

10 continue

else

$\mathrm{D}($ idir $)=\mathrm{B}($ eqnum(idir,inode $))$

endif

displ(inode, idir $)=D($ idir $)$

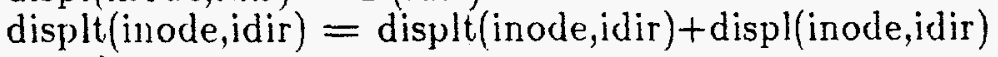

20 continue 


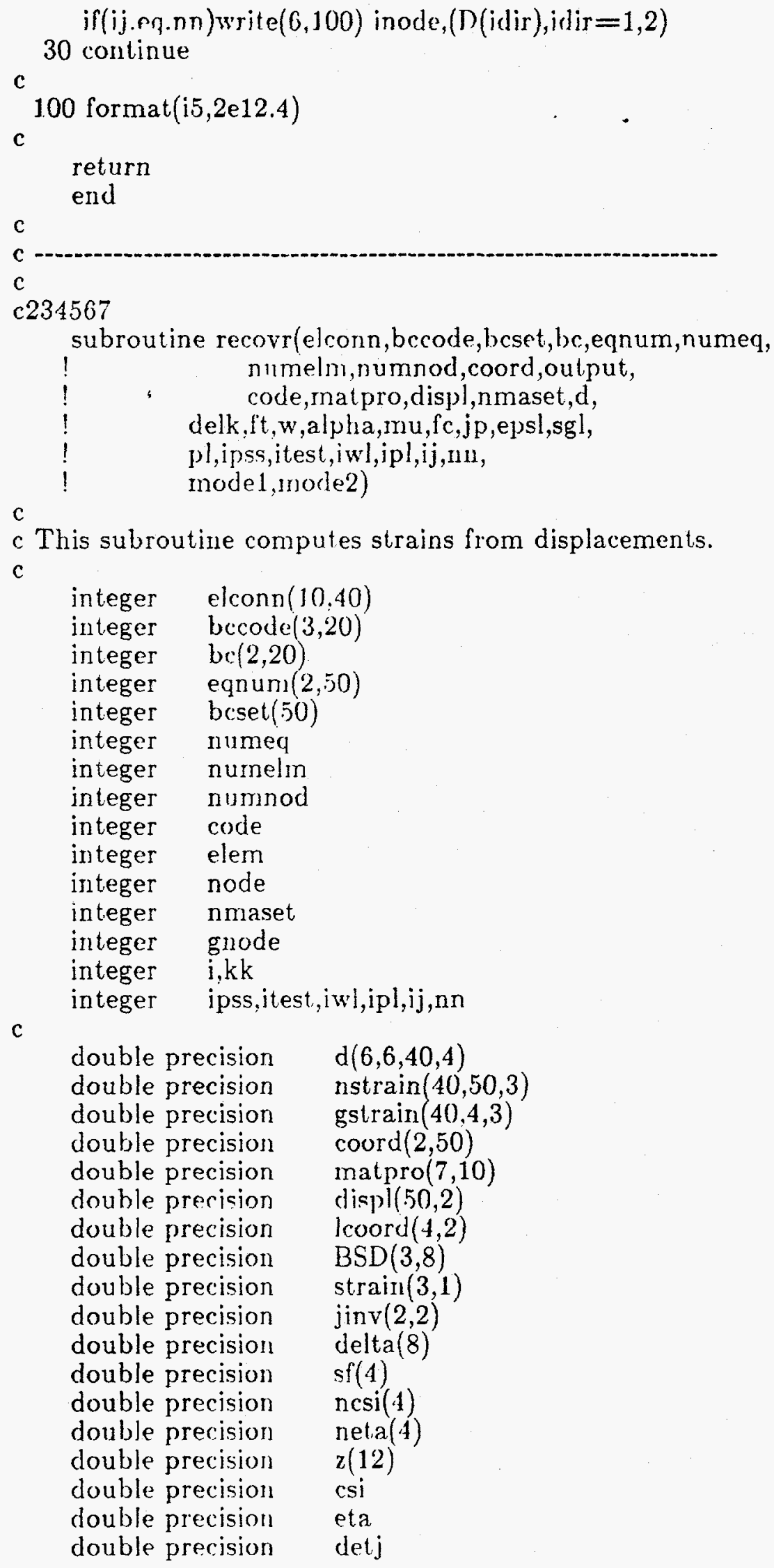




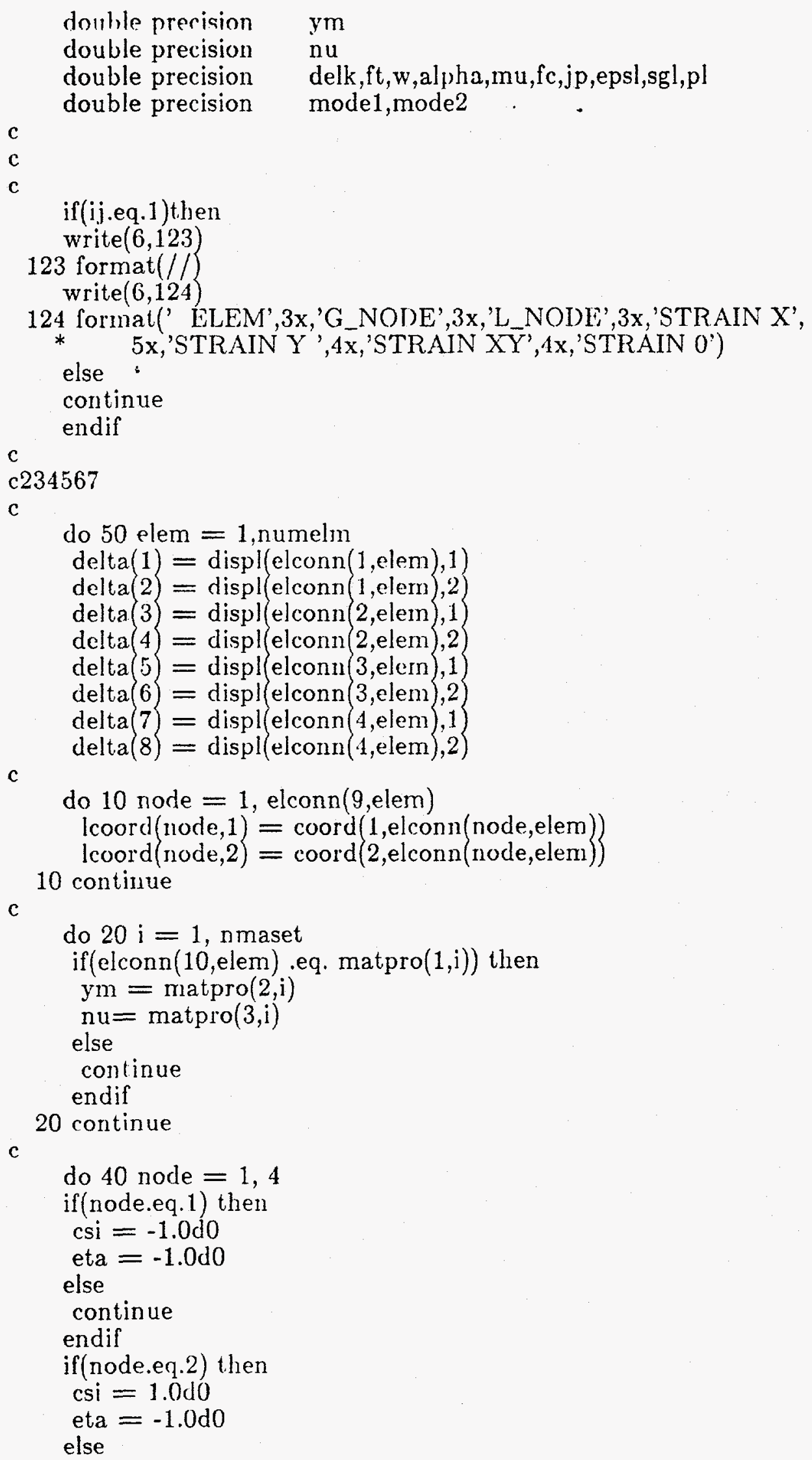




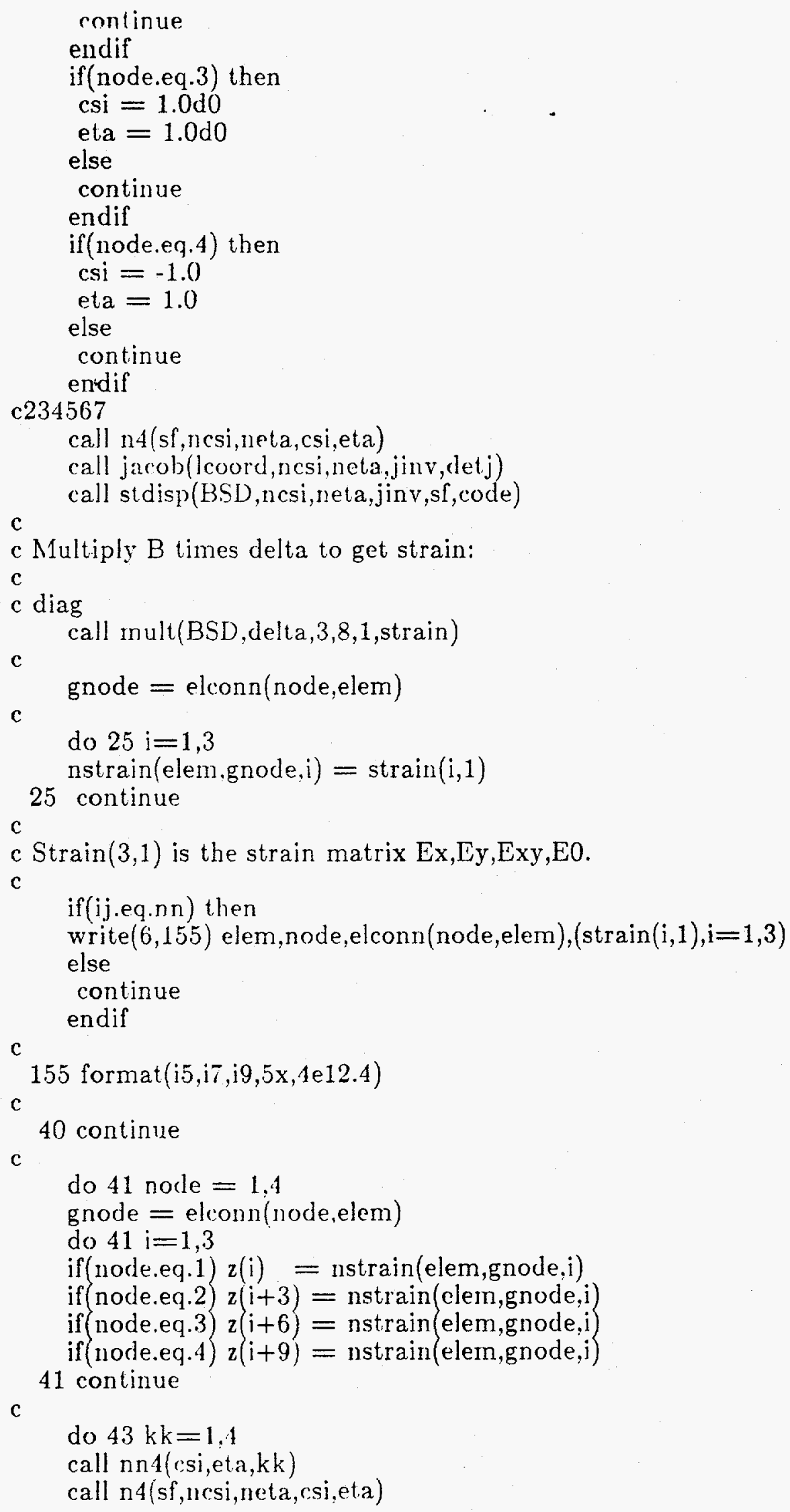




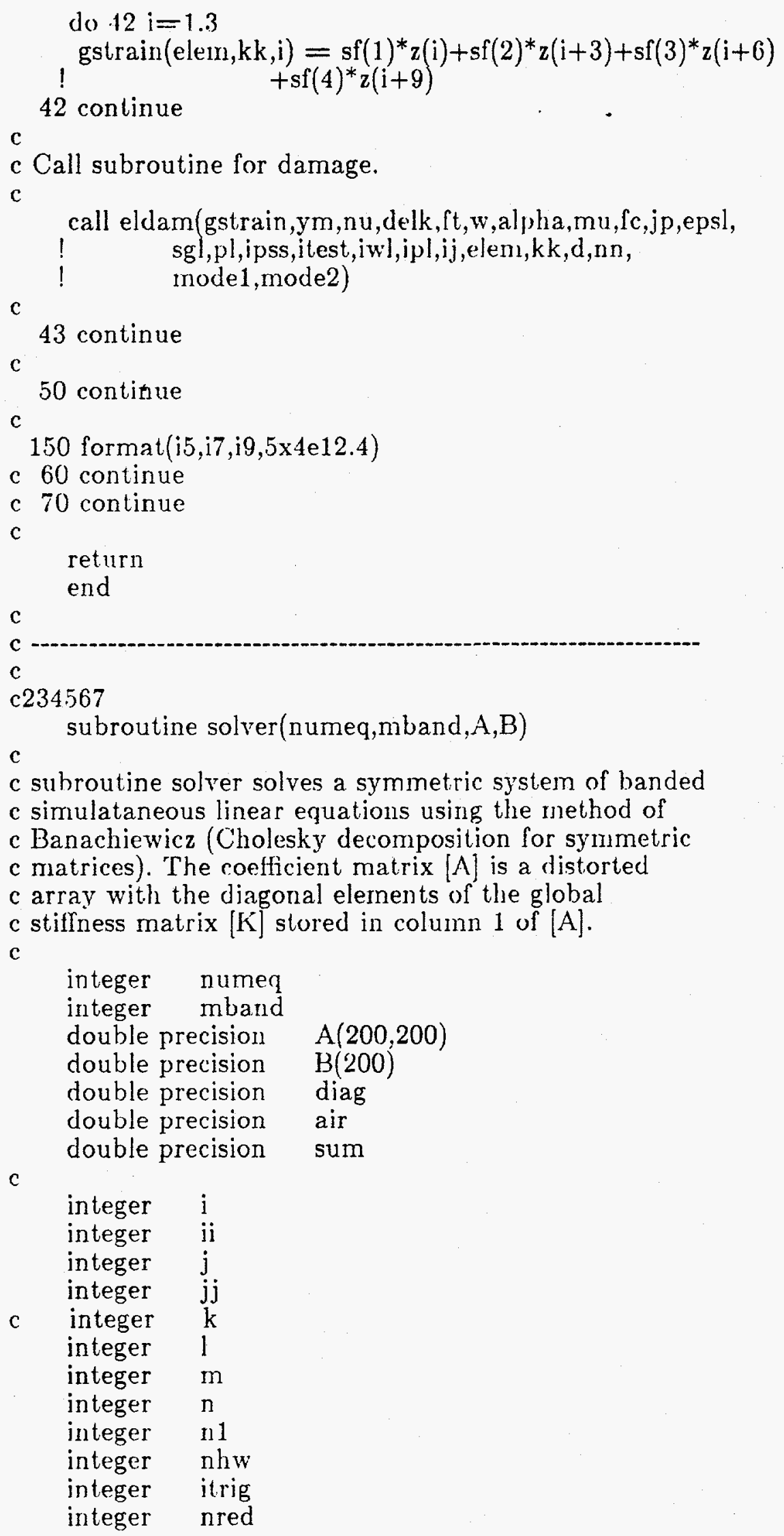




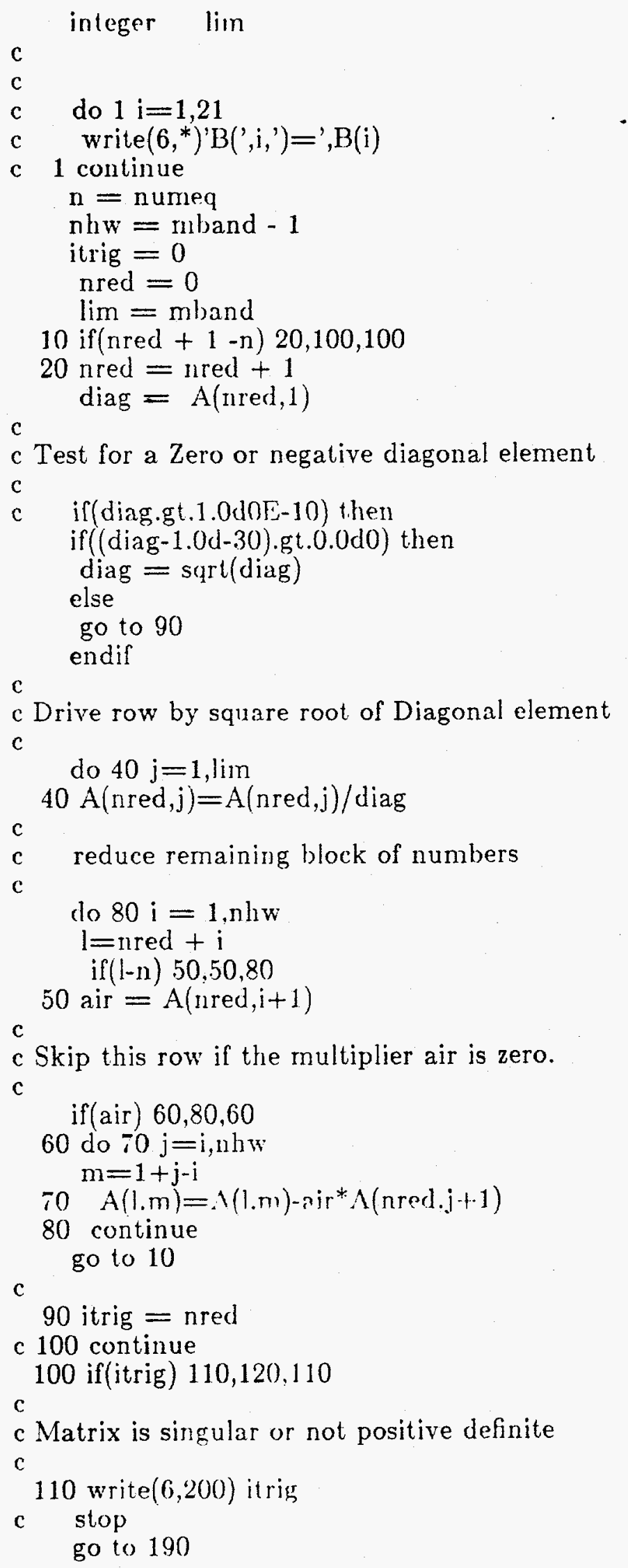




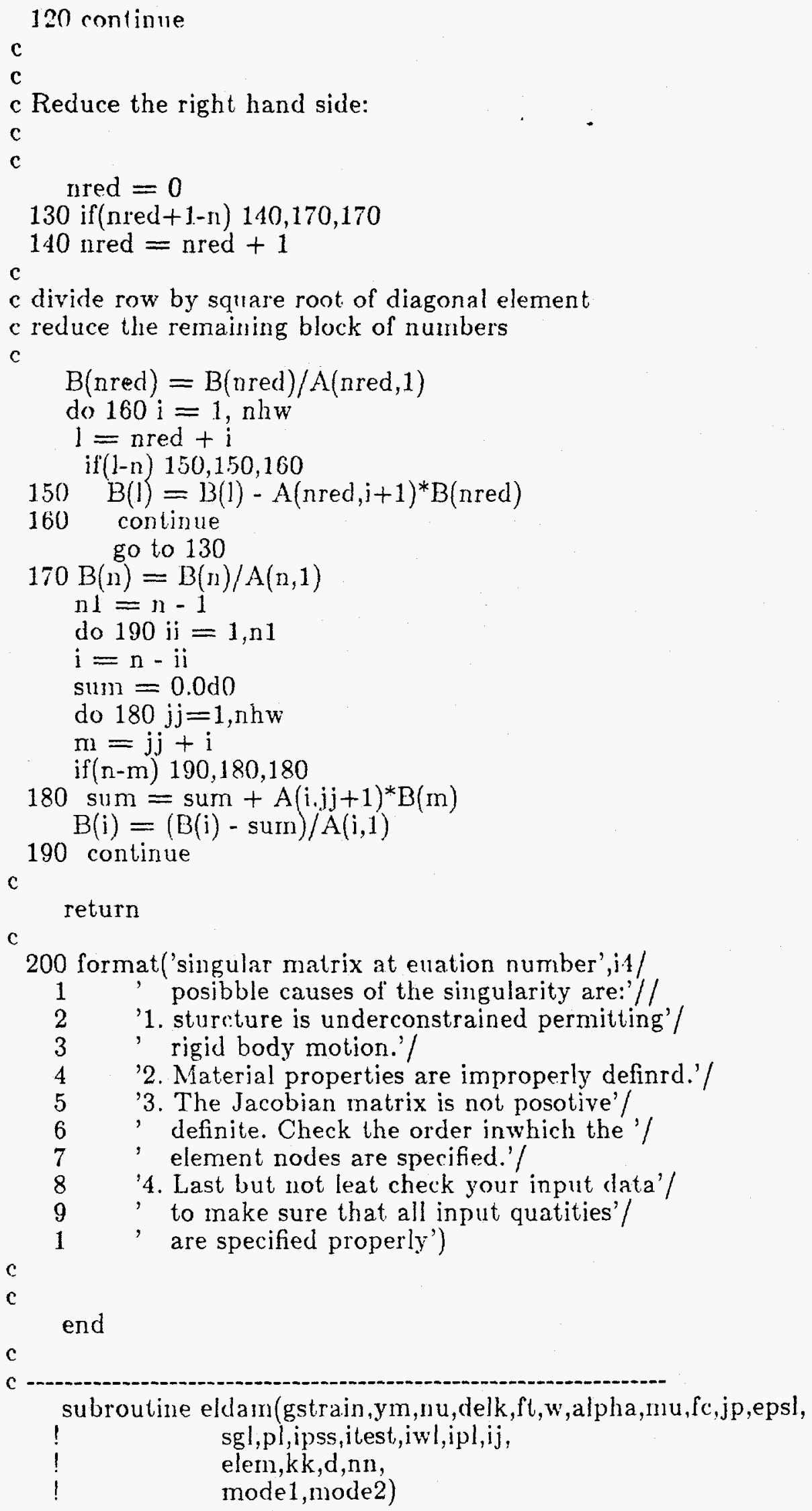


C

double precision $F(6,6,40,4), \operatorname{cc}(6,6,40,4), \operatorname{cc} 1(6,6,40,4)$,

$! \quad \operatorname{cc} 2(6,6,40,4), \mathrm{d}(6,6,40,4)$

double precision $\mathrm{sg} 11(40,4), \mathrm{sg} 22(40,4)$,

! $\quad \operatorname{sg} 33(40,4), \mathrm{sg} 12(40,4), \mathrm{p}, \mathrm{sgl}, \mathrm{pl}$

double precision gstrain(40,4,3), stt11, stt22,stt33,stt12,dstt11,

! dstt22, dstt33, dstt12,ste11,ste22,ste33,ste12,

sttvol

double precision $\mathrm{k}(40,4), \mathrm{t}(40,4), \mathrm{ft}$, delk,epsl

double precision std 11, std22, std 33, std 12

donble precision ci $(40,4), \mathrm{fc}, \mathrm{w}$, alpha,mu,ym,nu

double precision e1,e2,tg,pr1,pr2,pr3,pr 4

double precision mode1, mode2, mean

double precision vertor (3,3), eigen(3)

integer iswich,jp,iwl,ipl

integer ipss,itest,iter,ij,elem,kk

integer $\quad \mathrm{i}, \mathrm{j}, \mathrm{nn}$

open(unit $=3$,file='output',status $=$ 'old')

$\mathrm{c}$

c This subroutine sets the data for the damage relations.

c The part from here to 10 is used to initialize variables and c elastic matrices. Normally one goes directly to 10 .

c

$$
\begin{aligned}
& \text { if(ij.gt.1) go to } 10 \\
& \text { iter }=0 \\
& \text { iswich }=0 \\
& \text { stt11 }=0 . \mathrm{d} 0 \\
& \text { stt22 }=0.10 \\
& \mathrm{stt} 33=0 . \mathrm{d} 0 \\
& \text { stt } 12=0 . d 0 \\
& \text { sttvol }=0 . \mathrm{d} 0 \\
& \operatorname{std} 11=0 . \mathrm{d} 0 \\
& \text { std } 22=0 . \mathrm{d} 0 \\
& \operatorname{std} 33=0 . \mathrm{d} 0 \\
& \operatorname{std} 12=0 . \mathrm{do} \\
& \text { ste11 }=0 . \mathrm{d} 0 \\
& \text { ste } 22=0 . \mathrm{d} 0 \\
& \text { ste } 3.3=0 . \mathrm{d} 0 \\
& \text { ste12 }=0 . \mathrm{d} 0 \\
& \mathrm{p}=0 . \mathrm{d} 0 \\
& \mathrm{pl}=0 . \mathrm{d} 0 \\
& \mathrm{t}(\mathrm{elem}, \mathrm{kk})=0 . \mathrm{d} 0 \\
& \mathrm{k}(\mathrm{elem}, \mathrm{kk})=0 . \mathrm{d} 0 \\
& \text { ci }(\text { elem, } k \mathrm{k})=0 . \mathrm{d} 0 \\
& \mathrm{sg} 11 \text { (elem, kk) }=0 . \mathrm{d} 0 \\
& \text { sg22(elem,kk) }=0 . \mathrm{d} 0 \\
& \text { sg33 elem, } \mathrm{kk}=0 . \mathrm{d} 0 \\
& \mathrm{sg} 12 \text { elem, } \mathrm{kk})=0 . \mathrm{d} 0 \\
& \text { do } 150 \mathrm{i}=1,6 \\
& \text { do } 140 \mathrm{j}=1,6 \\
& F(i, j, \text { elem, } k \mathrm{k})=0 \text {. } \\
& \operatorname{cc}(i, j, \text { elem, kk })=0 \text {. } \\
& \mathrm{d}(\mathrm{i}, \mathrm{j}, \text { elem, } \mathrm{kk})=0 \text {. }
\end{aligned}
$$


1 in rontinue

150 continue

c iter is the iteration count.

c stt11, stt22, stt33 are the total strains in 1-1, 2-2, and 3-3 directions.

c std11, std22, std33 are the total damage strains in the three directions.

c ste11, ste22, ste33 are the total elastic strain in the three directions.

c $p$ is the pressure, $\mathrm{pl}$ is a limiting value of pressure, $t$ is the critical

$c$ stress, $k$ and ci are the damage parameter and surface, respectively.

c sg11, sg22, and $\operatorname{sg} 33$ are the stresses in the three directions.

c $F$ is the current flexibility tensor, cc is the added flexibility, and

$c \mathrm{~d}$ denotes the current stiffness tensor.

$c$ mean is the trace of the incremental strain tensor.

$\mathrm{c}$

C

mean $=(\mathrm{dstt} 11+\mathrm{dstt} 22+\mathrm{dstt} 33) / 3.0 \mathrm{~d} 0$

pr $1=1 .-\mathrm{nu}$

$\operatorname{pr} 2=\mathrm{nu} / \mathrm{pr} 1$

pr3 $=1 .+\mathrm{nu}$

pr4 $=1 . /(\operatorname{pr} 3 *(1.0-2.0 * \mathrm{nu}))$

el $=y$ in $*$ pr $1 *$ pr4

$\mathrm{e} 2=\mathrm{ym}^{*} \mathrm{nu}^{*} \mathrm{pr}^{4}$

$\operatorname{tg}=y \mathrm{~m} / \mathrm{pr} 3$

$\mathrm{t}(\mathrm{elem}, \mathrm{kk})=0.0$

if(mean.gt.0.0) then

$\mathrm{e} 1=\operatorname{mode} 1 * \mathrm{e} 1$

$\mathrm{e} 2=\operatorname{mode} 1 * \mathrm{e} 2$

$\operatorname{tg}=$ mode $1 * \operatorname{tg}$

else

continue

endif

C

c Form elastic matrices for each element "elem", and Gauss pt. "k".

c

$\mathrm{d}(1,1, \mathrm{elem}, \mathrm{kk})=\mathrm{e} 1$

$\mathrm{d}(1,2, \mathrm{elem}, \mathrm{kk})=\mathrm{e} 2$

$\mathrm{d}(1,3$, elem, $\mathrm{kk})=\mathrm{e} 2$

$\mathrm{d}(2,1, \mathrm{elem}, \mathrm{kk})=\mathrm{e} 2$

$\mathrm{d}(2,2, \mathrm{elem}, \mathrm{kk})=\mathrm{e} 1$

$\mathrm{d}(2,3$, elem, $\mathrm{kk})=\mathrm{e} 2$

$\mathrm{d}(3,1, \mathrm{elem}, \mathrm{kk})=\mathrm{e} 2$

$\mathrm{d}(3,2, \mathrm{elem}, \mathrm{kk})=\mathrm{e} 2$

d $3,3, \mathrm{elem}, \mathrm{kk}=\mathrm{e} 1$

$\mathrm{d}(4,4, \mathrm{elem}, \mathrm{kk})=\mathrm{tg}$

$\mathrm{d}(5,5$, elem, $\mathrm{kk})=\mathrm{tg}$

$\mathrm{d}(6,6$, elem, $\mathrm{kk})=\mathrm{tg}$

c

$F(1,1$, elem, kk $)=1 . / \mathrm{ym}$

if(mean.gt.0.0) then

$\mathrm{F}(1,1$, elem, $\mathrm{kk})=\mathrm{F}(1,1$, elem, $\mathrm{kk}) /$ mode 1

else

continue

endif

$\mathrm{F}(1,2$,elem, kk $)=-n \mathrm{u} * \mathrm{~F}(1,1$, elem, $\mathrm{kk})$

$\mathrm{F}(1,3$,elem, $\mathrm{k})=\mathrm{F}(1,2$, elem, $\mathrm{kk})$ 


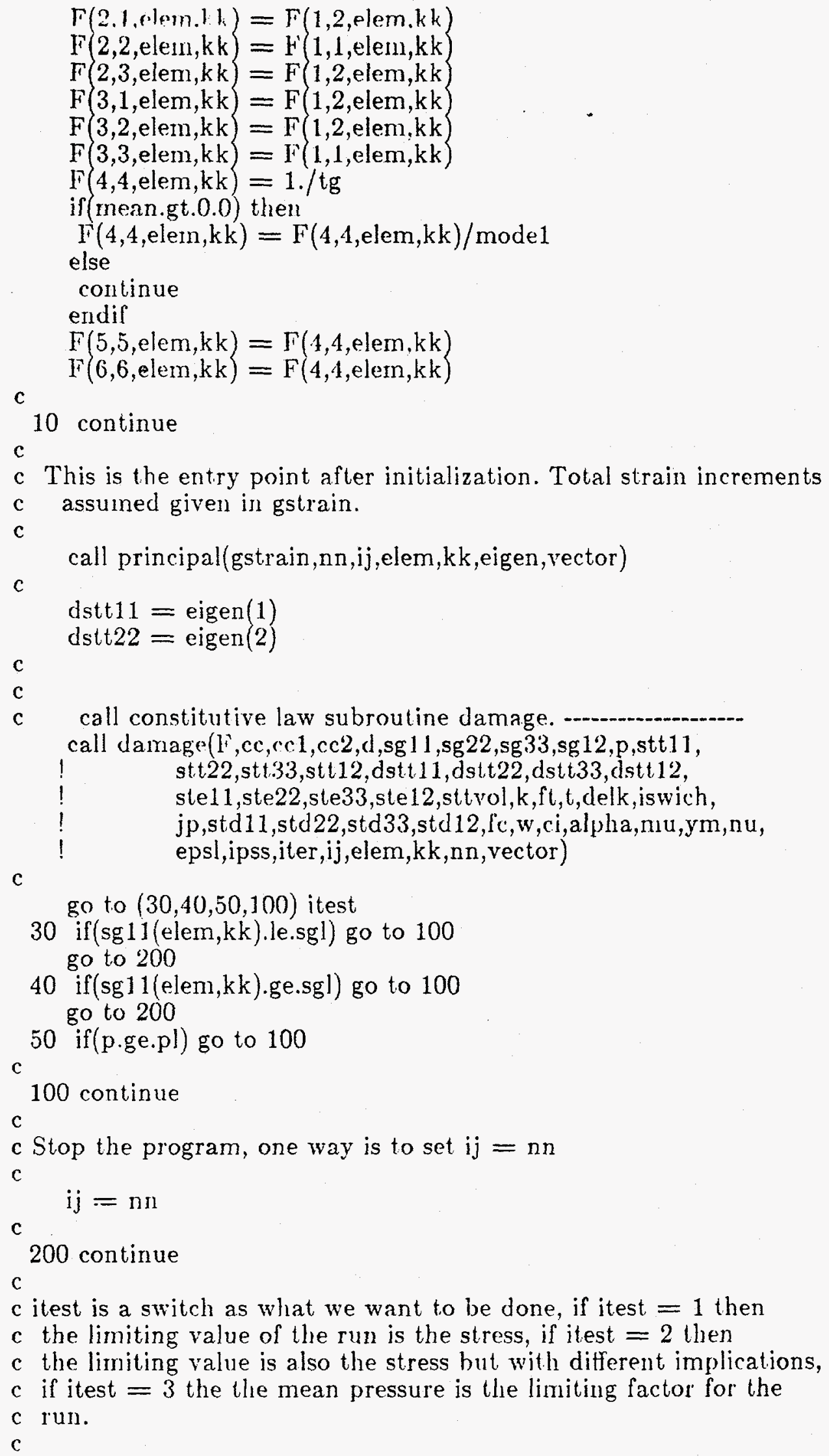


return

end

c

c-1-n-

c

subroutine damage(F,cc,cc1,cc2,d,sg11,sg22,sg33,sg 12,p,stt11, stt22,stt33,stt12,dstt11,dstt22,dstt33,dstt12, ste11,ste22,ste 33,ste12,st.tvol,k,ft,t,delk,iswich, jp,std11,std22,std33,std 12, fc,w,ci,alpha,mu,ym,nu, epsl,ipss,iel,iter,ij,elem,kk,nn,vector)

$c$

double precision $F(6,6,40,1), \operatorname{cc}(6,6,10,4), \operatorname{cc} 1(6,6,40,4)$,

! $\quad \operatorname{cc} 2(6,6,40,4), \mathrm{d}(6,6,40,4)$

double precision $\mathrm{sg} 11(40,4), \mathrm{sg} 22(40,4), \mathrm{sg} 33(40,4)$,

! $\quad \operatorname{sg} 12(40,4), \mathrm{p}$

double precision stt11,st, 22, stt 33 ,stt12,dstt11,
! dstt22,dstt33,dstt12,ste11,ste22,ste33,ste12,
! sitvol

double precision $\mathrm{k}(40,4), \mathrm{t}(40,4), \mathrm{ft}$, delk,epsl

double precision std11, std22,std33,std12

double precision ci $(40,4)$, fc, w, alpha, $m u, y m, n u$

double precision $\mathrm{sg} z 11, \mathrm{sg} z 22, \operatorname{sg} z 33, \operatorname{sgz} 12$

double precision sgnp11,sgnp22,sgnp33,sgnn11,sgnn22,sgnn33

double precision dedp11, dedp22, dedp33, dedn11, dedn22,dedn33

double precision dstd11,dstd22,dstd33,dstd12

double precision dste11,dste22, dste33,dste12

double precision $\mathrm{dn}, \mathrm{d} 21, \mathrm{~d} 31, \mathrm{ac} 21, \mathrm{ac} 31$

double precision tz,dsg11,dsg22,dsg $33, \mathrm{dsg} 12$

double precision trace 5 , trace 1 , trace 2 , trace 3

double precision sgp11,sgp22,sgp33,sgn11,sgn $22, \operatorname{sgn} 33$

double precision minsg,sge11,sge22,sge 33

double precision a11,a22,a33,a12,a13,a23

double precision ciz, second, third, fifth, $\mathrm{dkz}, \mathrm{dk}$

double precision aa $(3,3)$, ainv $(3,3)$

double precision sup11, sup22,sup33,sun11, sun22,sun33

double precision vector $(3,3)$, vectort $(3,3)$

double precision TR1 $(3,3), \operatorname{TR} 2(3,3)$

integer iswich,jp

integer ipss,iel,iter,ij,elem,kk

integer $\quad \mathrm{i}, \mathrm{j}$, iterm, $\mathrm{ji}$

integer iterz,nn

data iterm/10/,pib2/1.570796/

C

c initialize parameters for this step.

iter $=0$

$\mathrm{dk}=0$.

iter $z=$ iter

$\operatorname{sgz} 11=\operatorname{sg} 11$ (elem,kk)

sgz22 $=$ sg22(elem,kk)

$\mathrm{sgz} 33=\operatorname{sg} 33$ (elem,kk)

sgz12 $=$ sg12(elem,kk) 
C

$$
\begin{aligned}
& \operatorname{sgnp} 11=0 . d 0 \\
& \operatorname{sgn} 22=0 . d 0 \\
& \operatorname{sgnp} 33=0 . d 0 \\
& \operatorname{sgnn} 11=0 . d 0 \\
& \operatorname{sgnn} 22=0 . d 0 \\
& \operatorname{sgnn} 33=0 . d 0
\end{aligned}
$$

c

do $10 \mathrm{i}=1,6$

do $5 \mathrm{j}=1,6$

$\operatorname{cc} 1(\mathrm{i}, \mathrm{j}$, elem, $\mathrm{kk})=0$.

cc2 $(\mathrm{i}, \mathrm{j}, \mathrm{elem}, \mathrm{kk})=0$.

5 conlinue

10 continue

c

c

do $12 \mathrm{i}=1,3$

do $11 \mathrm{j}=1,3$

$\operatorname{vectort}(\mathrm{j}, \mathrm{i})=\operatorname{vector}(\mathrm{i}, \mathrm{j})$

11 continue

12 continue

c

c Assume step is elastic.

$$
\begin{aligned}
& \operatorname{dedp} 11=0 . d 0 \\
& \operatorname{dedp} 22=0 . d 0 \\
& \operatorname{dedp} 33=0 . d 0 \\
& \operatorname{dedn} 11=0 . d 0 \\
& \operatorname{dedn} 22=0 . d 0 \\
& \operatorname{dedn} 33=0 . d 0
\end{aligned}
$$

C

$$
\begin{aligned}
& \mathrm{dstd} 11=0 . \mathrm{d} 0 \\
& \mathrm{dstd} 22=0 . \mathrm{d} 0
\end{aligned}
$$$$
\mathrm{dstd} 33=0 . \mathrm{d} 0
$$$$
\mathrm{dstd} 12=0 . \mathrm{d} 0
$$

c

dste11 = dstt11

dste $22=\operatorname{dstt} 22$

dste $33=$ dstt 33

dste12 $=$ dstt12

c

c ipss is a path prescriber

$$
\begin{aligned}
& \text { c if ipss }=1 \text {-..- uniaxial stress, } \\
& \text { c if ipss }=2 \text {-.-.- biaxial stress, } \\
& \text { c if ipss }=3 \text {---- general stress path, } \\
& \text { c if ipss }=4 \text {--.- user prescribed palt. } \\
& \text { if(ipss.ne.4) go to } 110 \\
& \mathrm{dn}=1.0-11 \mathrm{u}^{*}(\mathrm{ac} 21+\mathrm{ac} 31) \\
& \mathrm{d} 21=(\operatorname{ac} 21-\mathrm{nu} *(1.0+\mathrm{ac} 31)) / \mathrm{dn} \\
& \mathrm{d} 31=(\operatorname{ac} 21-\mathrm{nu} *(1.0+\mathrm{ac} 21)) / \mathrm{dn} \\
& \text { dste } 22=\mathrm{d} 21 * \text { dste } 11 \\
& \text { dste } 33=\mathrm{d} 31 * \text { dste } 11 \\
& \text { go to } 150
\end{aligned}
$$




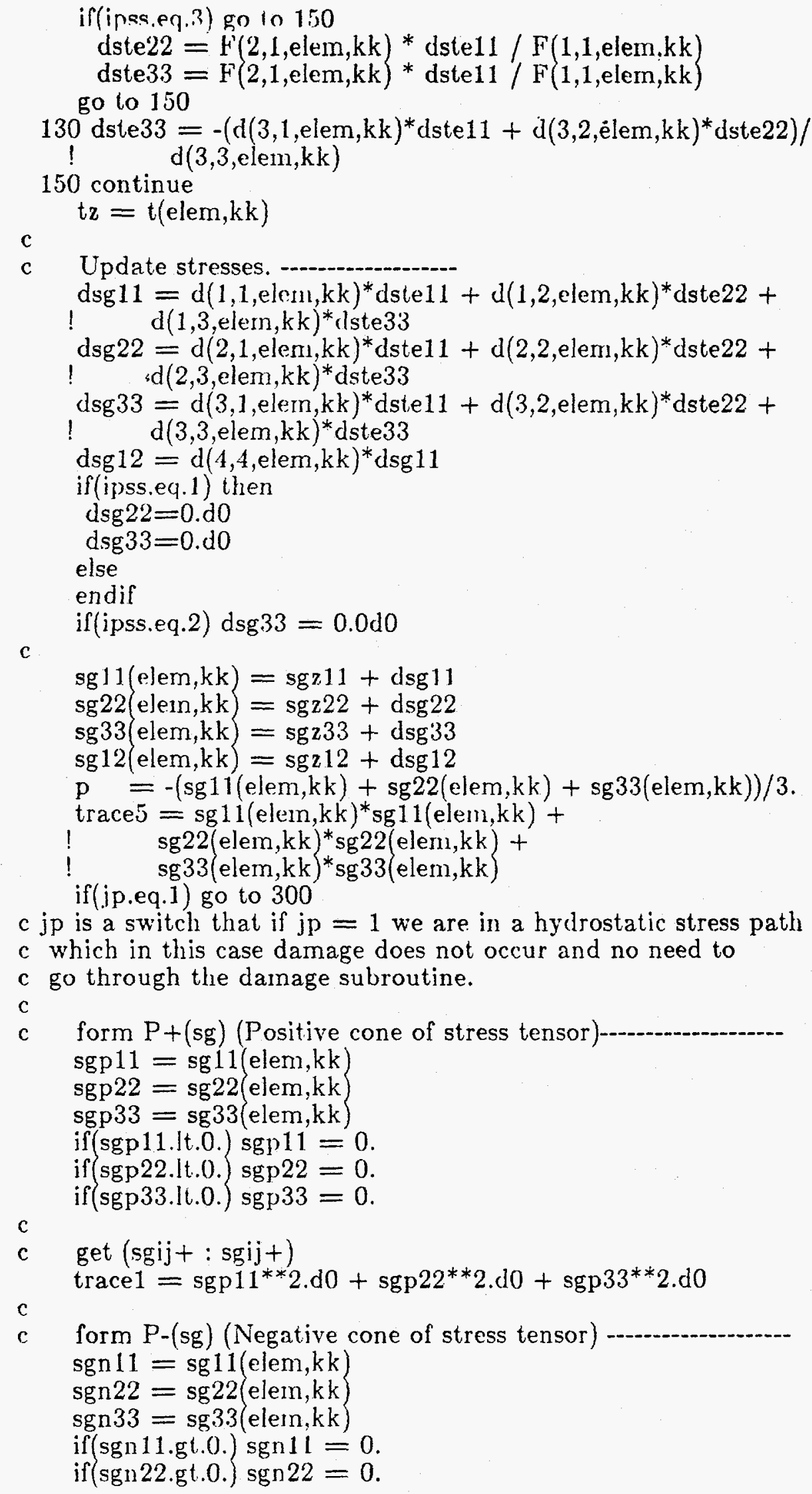


c determine the min. eigenvalue of the sgn tensor

minsg $=$ dabs(sgn11)

if (dabs(sgn 22$).$ lt.dabs $(\operatorname{sgn} 11))$ then

minsg $=$ dabs $(\operatorname{sgn} 22)$

if(dabs(sgn33).lt.dabs(sgn22))minsg $=$ dabs(sgn33)

continue

else

if(dabs(sgn33).lt.dabs(sgn11)) minsg $=\operatorname{dabs}(\operatorname{sgn} 33)$

endif

$\mathrm{c}$

$\mathrm{c}$

c

$\mathrm{c}$

c form the fourth order damage tensor $\mathrm{R}$ (sigma minus)

a $11=$ sge $11^{*}$ sge 11

a $22=$ sge $22 *$ sge 22

a.33 $=$ sge $33^{*}$ sge 3.3

a.12 = sge11*sge 22

a $13=$ sge $11 *$ sge 33

a23 $=$ sge $22 *$ sge 33

c

trace $3=\left(\operatorname{sgn} 11^{*} \mathrm{a} 11^{*} \operatorname{sgn} 11\right)+\left(\operatorname{sgn} 22^{*} \mathrm{a} 22^{*} \operatorname{sgn} 22\right)+\left(\operatorname{sgn} 33^{*} \mathrm{a} 33^{*} \operatorname{sgn} 33\right)+$

c

c

iter $=$ iter +1

$\mathrm{ciz}=\mathrm{ci}(\mathrm{elem}, \mathrm{kk})$

c

c

evaluate the damage surface.

if(trace2 .1t. .0000001) then

second $=0 . \mathrm{do}$

third $=0 . \mathrm{d} 0$

fifth $=0 . \mathrm{d} 0$

else

second $=w^{*}($ trare 3$) /$ trare 2

third $=w^{*}($ alpha $) * 9 . d 0 *(p * p)$

fifth $=\mathrm{w}^{*} \mathrm{mu}^{*}$ alpha*trace5

endif

c

ci(elem,kk $)=($ trace $1+$ second + fifth - third -

! $\left.\mathrm{tz}^{* *} 2 . \mathrm{d} 0\right)$

ci $($ elem, $\mathrm{kk})=0.5 \mathrm{~d} 0 *$ ci(elem, $\mathrm{kk})$

c $\quad$ ci(elem, $\mathrm{kk})=-2.0 \mathrm{~d} 0$

c For linear elastic problems make ci a negative number as

c suggested above.

c

c check if the damage surface is reached. 
if(ci(nom,kk).gt.rpsl) go to 170

ir(iter .eq. 1) go to 250

if(ci(elem,kk) .gt. -epsl) go to 250

170 continue

iswich $=1$

$\mathrm{C}$

c compute $\mathrm{dk}$ using secant approximation.

$\mathrm{dkz}=\mathrm{dk}$

if(iter .eq. 1) $\mathrm{dk}=$ delk

if(iter eq. 1) go to 175

$\mathrm{d} \mathrm{k}=\mathrm{dkz}{ }^{*}$ ci(elem, kk)/(ciz - ci(clem,kk))

175 continue

c

c compule the critical stress t.

$\mathrm{k}(\mathrm{elem}, \mathrm{kk})=\mathrm{k}(\mathrm{elem}, \mathrm{kk})+\mathrm{dk}$

$\mathrm{t}($ elem, kk $)=\mathrm{ft}^{*}$

! $(\operatorname{dexp}(1 . \mathrm{d} 0))^{*} \mathrm{~d} \log \left(1 . \mathrm{d} 0+\mathrm{ym}^{*} \mathrm{k}(\mathrm{elem}, \mathrm{kk})\right) /\left(1 . \mathrm{d} 0+\mathrm{ym}^{*} \mathrm{k}(\mathrm{elem}, \mathrm{kk})\right)$

c compute the added flexibility tensors in modes I and II.

c

if(trace1 .le. .0001) go to 176

$\operatorname{cc} 1(1,1$, elem, $\mathrm{kk})=\mathrm{dk}^{*}\left(\operatorname{sgp} 11^{*} \operatorname{sgp} 11\right) /$ trace 1

cc1 1,2 ,elem, $k \mathrm{k})=\mathrm{dk}^{*}\left(\mathrm{sgp} 11^{*} \mathrm{sgp} 22\right) /$ trace 1

$\operatorname{cc} 1(1,3$,elem, $\mathrm{kk})=\mathrm{dk}^{*}\left(\mathrm{sgp} 11^{*} \mathrm{sgp} 33\right) /$ trace 1

$\operatorname{cc} 1(2,2$, elem, $\mathrm{kk})=\mathrm{dk} *\left(\mathrm{sgp} 22^{*} \mathrm{sgp} 22\right) /$ trace 1

$\operatorname{cc} 1(2,3$, elem, $\mathrm{kk})=\mathrm{dk} *\left(\operatorname{sgp} 22^{*} \operatorname{sgp} 33\right) /$ trace 1

cc1 13,3, elem, $\mathrm{kk})=\mathrm{dk}^{*}\left(\mathrm{sgp} 33^{*} \mathrm{sgp} 33\right) /$ trace 1

$\operatorname{cc} 1(2,1$, elem, $\mathrm{kk})=\operatorname{cc} 1(1,2$,elem, kk)

$\operatorname{cc} 1(3,1$, elem, $\mathrm{kk})=\operatorname{cc} 1(1,3$,elem, $\mathrm{kk})$

$\operatorname{cc} 1(3,2$, elem, $\mathrm{kk})=\operatorname{cc} 1(2,3$, elem, $\mathrm{k} k)$

go to 179

176 do $178 \mathrm{i}=1,6$

do $177 \mathrm{j}=1,6$

177 cc1(i,j,elem,kk) $=0$.

178 continue

179 continue

c

if(trace2 .le. 0.0001$)$ go to 192

cc2 $(1,1$, elem, $k \mathrm{k})=\mathrm{dk}^{*}\left(\mathrm{a} 11 /\right.$ trace $2+\mathrm{mu}^{*}$ alpha - alpha $)$

cc2 1,2 ,elem, $k \mathrm{k})=\mathrm{dk}^{*}(\mathrm{a} 12 /$ trace 2 - alpha)

cc2 1,3 ,elem,kk $=\mathrm{dk}^{*}(\mathrm{a} 13 /$ trace2 - alpha $)$

cc2 2 ,2,elem, $k \mathrm{k})=\mathrm{dk}^{*}$ a $22 /$ trace $2+\mathrm{mu}^{*}$ alpha - alpha $)$

$\operatorname{cc} 2(2,3$, elem, $k \mathrm{k})=\mathrm{dk} *(\mathrm{a} 23 /$ irace 2 - alpha)

$\operatorname{cc} 2(3,3$, elem, $\mathrm{kk})=\mathrm{dk}^{*}\left(\mathrm{a} 33 /\right.$ trace $2+\mathrm{mu}^{*}$ alpha - alpha $)$

$\operatorname{cc} 2(2,1$, elem, $\mathrm{kk})=\operatorname{cc} 2(1,2$, elem, $\mathrm{kk})$

cc2 3,1, elem, $k \mathrm{k})=\mathrm{cc} 2(1,3, \mathrm{elem}, \mathrm{kk})$

$\operatorname{cc} 2(3,2$, elem, $\mathrm{kk})=\operatorname{cc} 2(2,3, \mathrm{elem}, \mathrm{kk})$

go to 195

192 do $194 \mathrm{i}=1.6$

do $193 \mathrm{j}=1,6$

$193 \mathrm{cc} 2(\mathrm{i}, \mathrm{j}, \mathrm{elem}, \mathrm{kk})=0.0$

194 continue

195 continue

c compute the total added and the current flexibility tensors. -- 


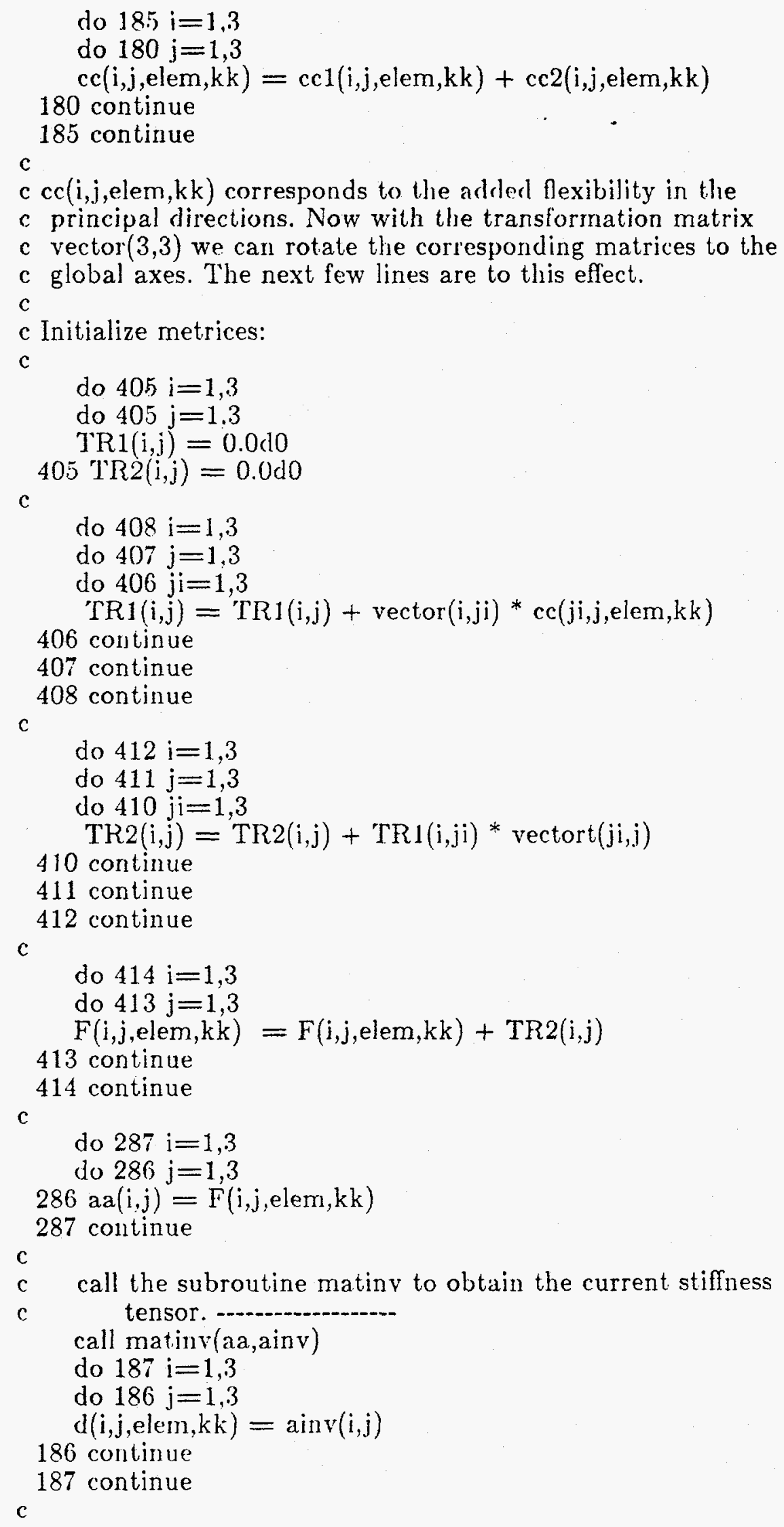


rompute the ineremental tamage strain.

sup11 $=\operatorname{cc} 1(1,1, \text { elem, kk })^{*} \operatorname{sgp} 11+\operatorname{cc} 1\left(1,2\right.$, elem,kk ${ }^{*} \operatorname{sgp} 22+$ $! \quad \operatorname{cc} 1(1,3$, elem, kk)*sgp33

$\sup 22=\operatorname{cc} 1(2,1, \text { elem,kk })^{*} \operatorname{sgp} 11+\operatorname{cc} 1(2,2, \text { elem,kk })^{*}$ sgp $22+$

! $\quad \operatorname{cc} 1(2,3, \text { elem,kk })^{*}$ sgp33

$\sup 33=\operatorname{cc} 1(3,1, \text { elem,kk })^{*} \operatorname{sgp} 11+\operatorname{cc} 1(3,2, \text { elem,kk })^{*} \operatorname{sgp} 22+$

$! \quad \operatorname{cc} 1(3,3, \text { elem,kk })^{*} \operatorname{sgp} 33$

sun $11=c c 2(1,1, \text { elem, kk })^{*}$ sg $11($ elem, kk $)+c c 2(1,2, \text { elem,kk })^{*}$

! $\quad \mathrm{sg} 22(\mathrm{elem}, \mathrm{kk})+\mathrm{cc} 2(1,3, \text { elern, kk) })^{*} \mathrm{sg} 33(\mathrm{elem}, \mathrm{kk})$

sun $22=\operatorname{cc} 2(2,1$, elem, kk)*sg11(elem,kk $)+\operatorname{cc} 2(2,2$,elem, kk)*

! $\quad \mathrm{sg} 22(\mathrm{elem}, \mathrm{kk})+\operatorname{cc} 2(2,3$, elem,kk)*sg33(elem,kk)

sun33 $=\operatorname{cc} 2(3,1, \mathrm{elem}, \mathrm{kk})^{*} \operatorname{sg} 11($ elem, $\mathrm{kk})+\operatorname{cc} 2(3,2, \mathrm{elem}, \mathrm{kk})^{*}$

C

$! \quad \mathrm{sg} 22(\mathrm{elem}, \mathrm{kk})+\operatorname{cc} 2(3,3, \mathrm{elem}, \mathrm{kk})^{*} \mathrm{sg} 33(\mathrm{elem}, \mathrm{kk})$

$\operatorname{ded} 11=\operatorname{dedp} 11+\sup 11$

$\operatorname{dedp} 22=\operatorname{dedp} 22+\sup 22$

dedp $33=\operatorname{dedp} 33+\sup 33$

$\operatorname{dedn} 11=\operatorname{dedn} 11+\operatorname{sun} 11$

$\operatorname{dedn} 22=\operatorname{dedn} 22+\operatorname{sum} 22$

$\operatorname{dedn} 33=\operatorname{dedn} 33+\operatorname{sun} 33$

$\mathrm{c}$

c compute the total damage strain increments.

$\operatorname{dstd} 11=\operatorname{dedp} 11+\operatorname{ded} 111$

$\operatorname{dstd} 22=\operatorname{dedp} 22+\operatorname{dedn} 22$

$\operatorname{dstd} 33=\operatorname{dedp} 33+\operatorname{ded} 133$

$\mathrm{c}$

c recompute the elastic strain increments.

dste11 = dste11 - sup 11 - sun 11

dste $22=$ dste $22-\sup 22-\operatorname{sun} 22$

dste33 = dste $33-\sup 33-\operatorname{sun} 33$

dste $12=$ dste 12

c

c adjust for the special conditions. -.-.-.-.-...-...-...

if(ipss.eq.4) go to 240

if(ipss.eq.3) go to 245

if(ipss.eq.2) go to 230

dste22 $=\mathrm{F}(2,1, \mathrm{elem}, \mathrm{kk}) *$ dste11/ $\mathrm{F}(1,1, \mathrm{elem}, \mathrm{kk})$

dste $33=$ dste 22

go to 245

230 dste $33=-\left(\mathrm{d}(3,1, \text { elem, kk })^{*}\right.$ dste $11+\mathrm{d}(3,2, \text { elem,kk })^{*}$ dste 22$) /$

go to 245

240 dste $22=\mathrm{d} 21 *$ dste 11

dste $33=\mathrm{d} 311^{*}$ dste 11

245 if(iter .le. iterm) go to 150

250 continue

c

c

c

updale strains.

c Note that for the particular problem of the shear wall

$c$ and due to the space limitations of the computer directory,

c total strains were not stored. Becuase of this the next

c few lines are commented out. If one desires to store total 
c strain also for reference, 2-rlimensional arrays slinuli be

c set up similar to the stress tensor, e.g. stdll(elem,kk),

c stt11(elem,kk).

c

c $\quad \operatorname{std} 11=\operatorname{std} 11+$ dstd 11

c $\quad \operatorname{std} 22=\operatorname{std} 22+\mathrm{dstd} 22$

c $\quad$ std $33=\operatorname{std} 33+$ dstd 33

c $\quad$ std $12=\operatorname{std} 12+\operatorname{dstd12}$

c

c

300 continue

c

c $\quad$ ste $11=$ stee $11+$ dste 11

c $\quad$ ste $22^{\circ}=$ ste $22+$ dste 22

c $\quad$ ste $33=$ ste $33+$ dste 33

c $\quad$ ste $12=$ ste $12+$ dste 12

c

c

c

dstt11 = dste11 + dst.d11

dstt $22=$ dste $22+$ dstd 22

dstt $33=$ dste $33+$ dstd 33

dstt12 $=$ dste $12+\mathrm{dstd} 12$

stt11 $=\operatorname{stt11}+\mathrm{dstt11}$

stt22 $=$ stt22 + dstt22

stt33 $=$ stt33 + dstt33

stt $12=\operatorname{stt} 12+\mathrm{dst} 12$

sttvol $=$ stt11 + stt $22+\operatorname{stt} 33$

return

end

c

$\mathrm{c}$

c

c

subroutine matinv(aa,ainv)

c

c This subroutine inverts a given matrix and stores it in ainv.

c

c

double precision aa $(3,3), \operatorname{ainv}(3,3)$

double precision $b(5,10)$

double precision temp

integer $\mathrm{n}, \mathrm{i}, \mathrm{j}, \mathrm{j} 1, \mathrm{j} 2, \mathrm{k}, \mathrm{kp} 1, \mathrm{l}, \mathrm{km} 1, \mathrm{j} 2$

$\mathrm{n}=3$

c

do $1 \mathrm{i}=1, \mathrm{n}$

do $1 \mathrm{j}=1, \mathrm{n}$

$1 \mathrm{~b}(\mathrm{i}, \mathrm{j})=\mathrm{aa}(\mathrm{i}, \mathrm{j})$

c load right half of matrix $b$ with unit matrix.

$\mathrm{j} 1=\mathrm{n}+1$

$\mathrm{j} 2=2 * \mathrm{n}$

do $2 \mathrm{i}=1, \mathrm{n}$

do $2 \mathrm{j}=\mathrm{j} 1, \mathrm{j} 2$

$2 \mathrm{~b}(\mathrm{i}, \mathrm{j})=0 . \mathrm{d} 0$ 


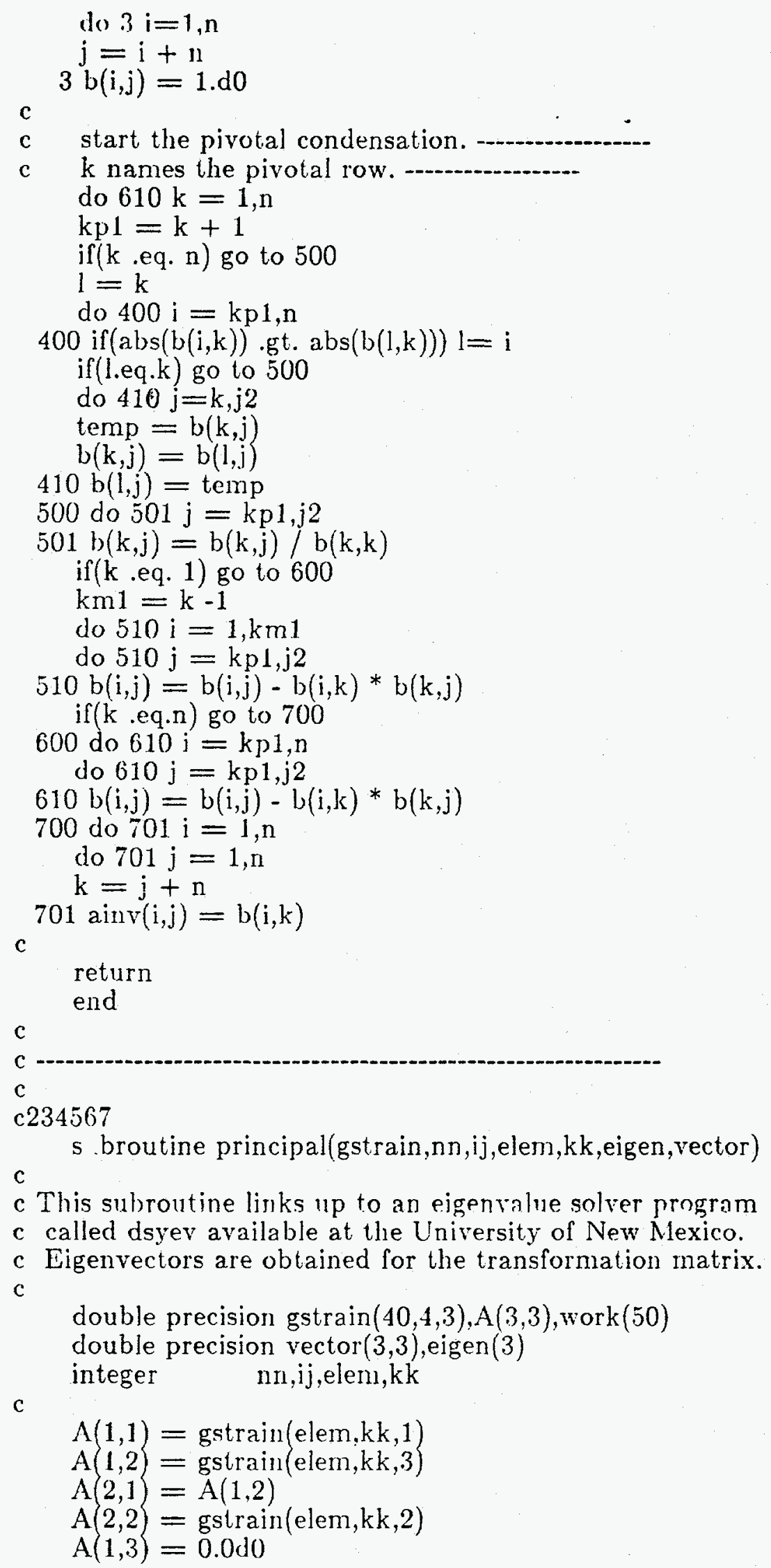


$\Lambda(2 . .9)=0.0 .10$

$\mathrm{A}(3,1)=\mathrm{A}(1,3)$

$A(3,2)=A(2,3)$

$\mathrm{A}(3,3)=-1.0$

c

call dsyev $(A, 3,3$,eigen, vector,work, 1 ,info $)$

if(info.ne. 0$)$ write $\left(6,{ }^{*}\right)$ 'dsyev did not converge:

$\mathrm{c}$

return

end

c

c

$\mathrm{c}$

c234567

subroutine principal1(A,eigen 1,numeq,mass,numelm)

c

c This subroutine links up with dsyev for the determination

c of the minimum eigenvalue problem.

c

double precision $\mathrm{SS}(200,200), \mathrm{A}(200,200)$

double precision vector $1(200,200)$, eigen $1(200)$

double precision work (200), nass

integer $\quad \mathrm{i}, \mathrm{j}$, numeq, numelm

integer $\quad 1,11$,info

$c$

do $11 \mathrm{i}=1$,numeq

$\mathrm{ll}=\mathrm{i}-1$

do $10 \mathrm{j}=\mathrm{i}$,numeq

$1=\mathrm{j}-11$

$S S(i, j)=A(i, 1)$

$S S(j, i)=S S(i, j)$

if(i.eq.j) then

$S S(i, j)=S S(i, j) *$ mass $/$ numelm

else

continue

endif

10 continue

11 continue

c

call dsyev(SS,numeq,numeq, eigen 1, vector 1, work,0,info)

c

retiurn

end

c

c 